\title{
Linear Quadratic Gaussian Balancing for Discrete-Time Infinite-Dimensional Linear Systems
}

\author{
Mark R. Opmeer and Ruth F. Curtain \\ SIAM J. Control Optim., vol. 43 (2004), no. 4, pp 1196-1221
}

\begin{abstract}
In this paper, we study the existence of linear quadratic Gaussian (LQG)-balanced realizations for discrete-time infinite-dimensional systems. LQG-balanced realizations are those for which the smallest nonnegative self-adjoint solutions of the control and filter Riccati equations are equal. We show that the control (filter) Riccati equation has a nonnegative self-adjoint solution if and only if the system is output (input) stabilizable. Our main result is that the transfer function of a discrete-time linear system has an approximately controllable and observable LQG-balanced realization iff it has an input and output stabilizable realization. The corresponding control and filter Riccati equations have unique nonnegative self-adjoint solutions. Moreover, approximately controllable and observable LQG-balanced realizations are unique up to a unitary state-space transformation. Finally, we show that the spectrum of the product of the smallest nonnegative self-adjoint solutions of the control and filter Riccati equations is independent of the particular realization.
\end{abstract}

\section{Introduction}

Simple models are normally preferred over complex ones in control systems design. Sometimes it is obvious how to construct a simple model for a physical system, but sometimes the characteristics essential to the controller design of a physical system are not obvious. One way to obtain a simple model in this last case is to first obtain a sophisticated model that takes into account every aspect that could be of interest and then perform model reduction on this sophisticated model. A simple model reduction procedure was introduced by Moore [7] and is now a textbook subject (see, e.g., Zhou and Doyle [17, Chapter 7]). The method proposed by Moore consists of truncating a balanced realization. A balanced realization (also called Lyapunov-balanced or internally balanced) is a realization for which the controllability and observability gramians are equal and diagonal. This procedure is applicable only to stable systems. Alternatively for unstable systems one can use truncations of a linear quadratic Gaussian 
(LQG)-balanced realization, which for rational transfer functions always exists. A LQG-balanced realization is a realization for which the smallest nonnegative self-adjoint solutions of the standard LQG control and filter Riccati equations are equal and diagonal. This method was proposed by Verriest [13], [14] and further developed by Jonckheere and Silverman [5]. For an alternative treatment see Mustafa and Glover [8]. The discrete-time case was considered by Hoffmann, Prätzel-Wolters, and Zerz [4].

In the case that the system is infinite-dimensional, the model/controller approximation becomes essential. One would like to use the methods of balanced truncation and LQG-balanced truncation in this case, too. The existence of Lyapunov-balanced and LQG-balanced realizations for irrational transfer functions, however, is nontrivial. Sufficient conditions for the existence of Lyapunovbalanced realizations were proved by Young [15], [16]. The purpose of this article is to give necessary and sufficient conditions for the existence of LQG-balanced realizations for discrete-time infinite-dimensional systems.

The proof is based on the correspondence between the Riccati equations of the plant and the Lyapunov equations of a certain closed-loop system and the result of Young on the existence of Lyapunov-balanced realizations. Although discrete-time systems have bounded operators, a number of features make the infinite-dimensional case more complicated than the finite-dimensional case. One is that input and output stabilizability, the natural infinite-dimensional generalizations of stabilizability and detectability, are not sufficient to obtain unique solutions of the control Riccati equation. Another is that it is not a priori clear that the natural factorization generated by the closed-loop system is coprime. These uniqueness and coprime properties were key features in the finite-dimensional proofs. Consequently, we have been forced to develop different proofs, predominantly algebraic, to get around these complications. We exploit the factorization idea previously used by Meyer and Franklin [6] (see also Ober and McFarlane [9]) for the finite-dimensional continuous-time case.

This article is organized as follows. We begin by reviewing the known finitedimensional theory on discrete-time LQG-balancing in section 2. In section 3 we review the relevant theory of discrete-time infinite-dimensional systems and, in particular, the linear quadratic regulator problem for this class of systems. Some of our results appear to be new. The previous standard results (e.g., Halanay and Ionescu [3]) assume a type of "exponential" stabilizability that is too strong for our purposes. In section 4 we review the relevant theory on Lyapunov-balanced realizations for discrete-time infinite-dimensional systems. The key result on the connection between normalized factorizations and the linear quadratic regulator theory is developed in section 5 . In section 6 we define the LQG-characteristic values and show that they are system invariants. In section 7 we derive many algebraic relations between the solutions of the control and filter Riccati equations and the Lyapunov equations of the closed-loop system. The often tedious algebraic proofs are relegated to the appendix. Finally, all the results from the previous sections are linked up in section 8 to prove our main result: an input and output stabilizable discrete-time system possesses an approximately controllable and observable LQG-balanced realization. These 
realizations are unique up to a unitary state-space transformation. This represents an elegant generalization of the finite-dimensional theory under minimal assumptions.

\section{LQG-balanced realizations: The finite-dimensional case}

In this section we review some of the results on finite-dimensional LQG-balanced realizations. We consider systems of the form

$$
x_{n+1}=A x_{n}+B u_{n}, \quad x(0)=x_{0}, \quad y_{n}=C x_{n}+D u_{n}
$$

where $A, B, C, D$ are matrices of compatible dimensions. For simplicity we consider the linear quadratic regulator (LQR) problem for the cost functional

$$
J\left(x_{0}, u\right):=\sum_{n=0}^{\infty}\left\|u_{n}\right\|^{2}+\left\|y_{n}\right\|^{2},
$$

where $y$ is given in terms of $x_{0}$ and $u$ by (1). The LQR problem consists of finding for a given $x_{0}$ that $u$ for which $J\left(x_{0}, u\right)$ is minimal. As is well known, this problem has a unique solution when $(A, B, C, D)$ is minimal: the optimal input $u^{\text {min }}$ is given by the state feedback $u_{n}^{\min }=-\left(I+D^{*} D+B^{*} Q B\right)^{-1}\left(D^{*} C+\right.$ $\left.B^{*} Q A\right) x_{n}$, where $Q$ is the unique nonnegative solution of the Riccati equation

$$
A^{*} Q A-Q+C^{*} C=\left(C^{*} D+A^{*} Q B\right)\left(I+D^{*} D+B^{*} Q B\right)^{-1}\left(D^{*} C+B^{*} Q A\right),
$$

and the optimal cost is given by $J\left(x_{0}, u^{\min }\right)=\left\langle x_{0}, Q x_{0}\right\rangle$. By duality the optimal filter cost is given by $\left\langle x_{0}, P x_{0}\right\rangle$, where $P$ is the unique nonnegative solution of the Riccati equation

$$
A P A^{*}-P+B B^{*}=\left(B D^{*}+A P C^{*}\right)\left(I+D D^{*}+C P C^{*}\right)^{-1}\left(D B^{*}+C P A^{*}\right) .
$$

The quantity $\left\langle x_{0}, P x_{0}\right\rangle$ can be interpreted as a measure of the difficulty of reconstructing the initial state $x_{0}$ from noisy measurements. The eigenvalues of the product $P Q$ are similarity invariants. It was shown by Fuhrmann and Ober [2] that the square roots of the eigenvalues of $P Q$ (called the LQG-characteristic values) are the singular values of a certain Hankel operator associated with the system. These invariants can be interpreted as a measure of how important the subspace generated by the eigenvector is for the compensator design. This can be seen from the LQG-balanced realization. An LQG-balanced realization is a realization of the plant such that $P=Q=\Lambda$, where $\Lambda$ is the diagonal matrix containing the LQG-characteristic values. Let $\lambda_{i}$ be the square root of an eigenvalue of $P Q$ with eigenvector $x_{i}$ of length one. Then, in the LQG-balanced realization, the optimal cost with initial condition $x_{i}$ is $\lambda_{i}$ and the difficulty of reconstructing this initial state from noisy measurements is also $\lambda_{i}$. The idea behind LQG-balanced truncation is to restrict the system to the subspace 
generated by the eigenvectors corresponding to the largest eigenvalues. Since this subspace is most important for compensator design, the system obtained by LQG-balanced truncation seems to be a reasonable approximation. There is a bound on the distance between a plant and an LQG-balanced truncation of the plant in terms of the discarded LQG-characteristic values; see Mustafa and Glover [8, section 8.4.5].

The existence of LQG-balanced realizations in the finite-dimensional case is easily proved as follows:

1. Start with a minimal realization $(A, B, C, D)$ and compute the solutions $Q$ and $P$ of the Riccati equations.

2. Write $P^{1 / 2} Q P^{1 / 2}=U \Lambda^{2} U^{*}$ with $\Lambda$ diagonal.

3. Let $T:=Q^{1 / 2} U \Lambda^{-1 / 2}$.

Then it is easily seen that $\left(T A T^{-1}, T B, C T^{-1}, D\right)$ is an LQG-balanced realization.

In the infinite-dimensional case this proof no longer works. The main problem is that the singular value decomposition performed at Step 2 cannot always be made in the infinite-dimensional case. (One has to assume a compactness condition.) Even if it can, then usually the singular values form a sequence with zero as limit point and the operator $\Lambda^{-1 / 2}$ mentioned at the third step is unbounded. Because of this unboundedness, it is unclear whether the expressions $T A T^{-1}$ and $T B$ make sense. To avoid these problems we take a different approach, mentioned in the introduction: we use the known result for the existence of Lyapunov-balanced realizations. In this article we consider only the existence of LQG-balanced realizations. The study of the properties of truncated LQG-balanced realizations will be done elsewhere. In slight contrast with the definition above we will call a realization LQG-balanced if $P=Q$. We do not require that they are diagonal, since this is not always possible in infinite dimensions.

\section{Discrete-time infinite-dimensional systems}

In this section we review that part of the theory of discrete-time infinite-dimensional systems that we need in this article. Discrete-time infinite-dimensional systems have been treated in a number of texts (e.g., [1], [3], [12]). However, the standard treatments of the linear quadratic theory assume the strong concept of power stabilizability, i.e., the existence of an $F$ such that $\left\|(A+B F)^{n}\right\| \leq M \lambda^{n}$ for some constants $M>0,0<\lambda<1$ and all positive integers $n$. Unfortunately, this concept is not suitable for a nice theory of LQG-balanced realizations. Thus in this section we reexamine the basic concepts under weaker stabilizability assumptions.

A discrete-time infinite-dimensional system or simply a system is a quadruple of bounded operators $(A, B, C, D) \in \mathcal{L}(X) \times \mathcal{L}(U, X) \times \mathcal{L}(X, Y) \times \mathcal{L}(U, Y)$, where 
$X, U, Y$ are separable Hilbert spaces. For an input $u$ and initial condition $x_{0}$, the state $x$ and output $y$ of the system are defined by

$$
x_{n+1}=A x_{n}+B u_{n} \quad x(0)=x_{0} \quad y_{n}=C x_{n}+D u_{n} .
$$

The observability map $\mathcal{C}$ of a discrete-time system $(A, B, C, D)$ is defined by

$$
(\mathcal{C} x)_{i}:=C A^{i} x, \quad i \in \mathbb{N} .
$$

The discrete-time system $(A, B, C, D)$ is said to be approximately observable if $\operatorname{ker} \mathcal{C}=\{0\}$ (see Curtain and Zwart [1]). There are many generalizations of the finite-

dimensional concept of observability to an infinite-dimensional setting, and the concept of approximate observability is one. Our main use of approximate observability is that realizations may, without loss of generality, be assumed to be approximately controllable and observable. This may not always be the case for other generalizations of observability. Here, approximately controllable and observable plays the role that minimal plays in finite dimensions.

The discrete-time system $(A, B, C, D)$ is said to be output stable if the image of $\mathcal{C}$ is contained in $l^{2}(\mathbb{N} ; Y)$. The observability gramian $L_{C}$ of an output stable system is defined as $L_{C}:=\mathcal{C}^{*} \mathcal{C}$. We now give an alternative characterization of output stability in terms of solutions of a certain Lyapunov equation.

Lemma 3.1. Let $(A, B, C, D)$ be a discrete-time system. The following are equivalent statements:

1. The system is output stable.

2. The observation Lyapunov equation

$$
A^{*} L A-L+C^{*} C=0
$$

has a nonnegative self-adjoint solution.

If one (and hence both) of these hold, then the observability gramian is the smallest nonnegative self-adjoint solution of the observation Lyapunov equation.

Proof. We first show that 1 implies 2. It is easily seen that the observability gramian is given by the formula $L_{C}=\sum_{i=0}^{\infty} A^{* i} C^{*} C A^{i}$, and substituting this into the observation Lyapunov equation shows that it is a solution.

That 2 implies 1 is proved as follows. Suppose that the observation Lyapunov equation has a nonnegative self-adjoint solution $L$. Then multiplying (4) from the left with $A^{* n}$ and from the right with $A^{n}$ and summing from $n=0$ to $N$ gives

$$
\begin{aligned}
\sum_{n=0}^{N} A^{* n} C^{*} C A^{n} & =\sum_{n=0}^{N} A^{* n} L A^{n}-\sum_{n=0}^{N} A^{* n+1} L A^{n+1} \\
& =L-A^{* N+1} L A^{N+1} \leq L .
\end{aligned}
$$


Letting $N \rightarrow \infty$ shows that $L_{C}$ is a bounded map and so $\mathcal{C}$ is bounded. That $L_{C}$ is smaller than any other nonnegative self-adjoint solution of the observation Lyapunov equation is obvious from the above inequality.

The following result shows that strong stability implies the uniqueness of solutions of Lyapunov equations. We remind the reader that an operator $A$ is called strongly (or asymptotically) stable if for all $x \in X$ we have $A^{n} x \rightarrow 0$ as $n \rightarrow \infty$.

Lemma 3.2. Let $(A, B, C, D)$ be output stable and let $A$ be strongly stable. Then the observability gramian is the unique nonnegative self-adjoint solution of the observation Lyapunov equation (4).

Proof. According to Lemma 3.1 the observability gramian is a nonnegative selfadjoint solution of the observation Lyapunov equation, so we only have to show that it is the unique nonnegative self-adjoint solution. Let $L$ be a nonnegative self-adjoint solution of the observation Lyapunov equation. Then, as in the proof of Lemma 3.1, we have for all $N \in \mathbb{N}$

$$
\sum_{n=0}^{N} A^{* n} C^{*} C A^{n}=\sum_{n=0}^{N} A^{* n} L A^{n}-\sum_{n=0}^{N} A^{* n+1} L A^{n+1}=L-A^{* N+1} L A^{N+1} .
$$

We then have for all $x, y \in X$

$$
\left\langle\sum_{n=0}^{N} A^{* n} C^{*} C A^{n} x, y\right\rangle=\langle L x, y\rangle-\left\langle L A^{N+1} x, A^{N+1} y\right\rangle .
$$

Letting $N \rightarrow \infty$ and using that $A$ is strongly stable, we have for all $x, y \in X$

$$
\left\langle L_{C} x, y\right\rangle=\langle L x, y\rangle .
$$

This implies that $L=L_{C}$. Since $L$ was an arbitrary nonnegative self-adjoint solution, this implies that $L_{C}$ is the unique nonnegative self-adjoint solution of the observation Lyapunov equation.

A discrete-time system $(A, B, C, D)$ is called output stabilizable if there exists an $F \in \mathcal{L}(X, U)$ such that $(A+B F, 0,[F ; C+D F], 0)$ is output stable. (Note that we use the notation $[-;-]$ for a block column vector and $[-,-]$ for a block row vector.) Output stabilizability is a necessary and sufficient condition for the solvability of the LQR problem. To show this we first review some well-known results on the LQR problem in infinite dimensions. For a system $(A, B, C, D)$ with input, state, and output related by (2), we consider the cost functional

$$
J\left(x_{0}, u\right):=\sum_{n=0}^{\infty}\left\|u_{n}\right\|^{2}+\left\|y_{n}\right\|^{2} .
$$

The well-known linear quadratic regulator problem is as follows. Find a sequence $u^{\text {min }}$ such that $J\left(x_{0}, u^{\text {min }}\right) \leq J\left(x_{0}, u\right)$ for all sequences $u$. A system is said to 
satisfy the finite cost condition if for every initial state $x_{0}$ there exists a $u$ such that $J\left(x_{0}, u\right)<\infty$. Just as in the finite-dimensional case one can prove that if the finite cost condition is satisfied, then there exists a unique optimal control $u^{\text {min }}$; we actually have $J\left(x_{0}, u^{\min }\right)<J\left(x_{0}, u\right)$ for all other sequences $u$ and $J\left(x_{0}, u^{\min }\right)=\left\langle x_{0}, Q x_{0}\right\rangle$, where $Q$ is the smallest nonnegative self-adjoint solution of the control algebraic Riccati equation (CARE) associated with the system $(A, B, C, D)$,

$$
A^{*} Q A-Q+C^{*} C=\left(C^{*} D+A^{*} Q B\right)\left(S+B^{*} Q B\right)^{-1}\left(D^{*} C+B^{*} Q A\right),
$$

where $S:=I+D^{*} D$. Moreover, $u^{\text {min }}$ can be given by a state feedback. All of this can be found, for example, in Curtain and Zwart [1, Exercise 6.34]. The operator $Q$ is called the optimal cost operator. The following lemma gives conditions that are equivalent to the finite cost condition.

Lemma 3.3. The following statements about a discrete-time system $(A, B, C, D)$ are equivalent:

1. The discrete-time system is output stabilizable.

2. The discrete-time system satisfies the finite cost condition.

3. The CARE (5) of the discrete-time system has a nonnegative self-adjoint solution.

Proof. Suppose the discrete-time system is output stabilizable. Then there exists an $F$ such that $(A+B F, 0,[F ; C+D F], 0)$ is output stable; denote the observability map of this system by $\mathcal{C}_{F}$. Add the equation $u:=F x$ to (2). Call the solution $(u, x, y)$ of this set of equations $\bar{u}, \bar{x}, \bar{y}$. Then $[\bar{u}, \bar{y}]=\mathcal{C}_{F} x_{0}$ and since $\mathcal{C}_{F}$ is bounded we see that $[\bar{u}, \bar{y}]$ has finite $l^{2}$ norm. That is, $J\left(x_{0}, \bar{u}\right)<\infty$ and the system satisfies the finite cost condition.

An outline of the proof that 2 implies 3 can be found in Curtain and Zwart [1, Exercise 6.34].

For 3 implies 1, we will show that the feedback $F:=-\left(B^{*} Q B+I+\right.$ $\left.D^{*} D\right)^{-1}\left(D^{*} C+B^{*} Q A\right)$, where $Q$ is a solution of the CARE (5), is output stabilizing. We will do this by showing that $Q$ is a solution of the observation Lyapunov equation of the system $(A+B F, 0,[F ; C+D F], 0)$. We want to show that $Q$ satisfies

$$
(A+B F)^{*} Q(A+B F)-Q+\left[F^{*}, C^{*}+F^{*} D^{*}\right]\left[\begin{array}{c}
F \\
C+D F
\end{array}\right]=0 .
$$

This is equivalent to

$$
\begin{aligned}
A^{*} Q A & -Q+C^{*} C+F^{*}\left(B^{*} Q B+I+D^{*} D\right) F \\
& +F^{*}\left(B^{*} Q A+D^{*} C\right)+\left(A^{*} Q B+C^{*} D\right) F=0 .
\end{aligned}
$$

Substituting for $F$ in (6) we obtain

$$
A^{*} Q A-Q+C^{*} C=\left(C^{*} D+A^{*} Q B\right)\left(S+B^{*} Q B\right)^{-1}\left(D^{*} C+B^{*} Q A\right),
$$

which is precisely CARE (5). 
The controllability map $\mathcal{B}$ of a discrete-time system $(A, B, C, D)$ is defined for finitely nonzero $U$-valued sequences $u$ by

$$
\mathcal{B} u:=\sum_{i=0}^{\infty} A^{i} B u_{-i-1} .
$$

The discrete-time system $(A, B, C, D)$ is said to be approximately controllable if ker $\mathcal{B}^{*}=\{0\}$. The discrete-time system $(A, B, C, D)$ is said to be input stable if $\mathcal{B}$ extends to a bounded map from $l^{2}\left(\mathbb{Z}^{-} ; U\right)$ to $X$. The controllability gramian $L_{B}$ of an input stable system is defined as $L_{B}:=\mathcal{B B}^{*}$. A discrete-time system $(A, B, C, D)$ is called input stabilizable if there exists an $L \in \mathcal{L}(Y, X)$ such that $(A+L C,[L, B+L D], 0,0)$ is input stable. The following dual results of the results proven earlier hold.

Lemma 3.4. Let $(A, B, C, D)$ be a discrete-time system. The following are equivalent statements:

1. The system is input stable.

2. The control Lyapunov equation

$$
A L A^{*}-L+B B^{*}=0
$$

has a nonnegative self-adjoint solution.

If one (and hence both) of the above holds, then the controllability gramian is the smallest nonnegative self-adjoint solution of the control Lyapunov equation.

Lemma 3.5. Let $(A, B, C, D)$ be input stable and let $A^{*}$ be strongly stable. Then the controllability gramian is the unique nonnegative self-adjoint solution of the control Lyapunov equation (8).

Lemma 3.6. The following statements about a discrete-time system $(A, B, C, D)$ are equivalent:

1. The discrete-time system is input stabilizable.

2. The dual system $\left(A^{*}, C^{*}, B^{*}, D^{*}\right)$ satisfies the finite cost condition.

3. The filter algebraic Riccati equation (FARE)

$$
A P A^{*}-P+B B^{*}=\left(B D^{*}+A P C^{*}\right)\left(R+C P C^{*}\right)^{-1}\left(D B^{*}+C P A^{*}\right),
$$

where $R:=I+D D^{*}$, of the discrete-time system has a nonnegative selfadjoint solution.

We now give a condition under which CARE (5) has a unique nonnegative self-adjoint solution.

Lemma 3.7. Let $(A, B, C, D)$ be an input and output stabilizable discrete-time system. Let $Q$ be a nonnegative self-adjoint solution of the CARE (5), and assume that $A_{Q}:=A-B\left(S+B^{*} Q B\right)^{-1}\left(D^{*} C+B^{*} Q A\right)$ is strongly stable. Then $Q$ is the unique nonnegative self-adjoint solution of CARE (5). 
Proof. For the proof we need the following algebraic relations, which are proved in the appendix (Lemmas 10.3 and 10.4). Suppose $Q_{1}$ and $P_{1}$ are nonnegative self-adjoint solutions of the CARE and FARE, respectively, and define $A_{Q_{1}}$ similar to $A_{Q}$ above and $A_{P_{1}}:=A-\left(B D^{*}+A P_{1} C^{*}\right)\left(R+C P_{1} C^{*}\right)^{-1} C$. Then the following relation holds:

$$
\left(I+P_{1} Q_{1}\right) A_{Q_{1}}=A_{P_{1}}\left(I+P_{1} Q_{1}\right) .
$$

The following algebraic relation is also proven in the appendix (Lemma 10.4). If $Q_{1}$ and $Q_{2}$ are nonnegative self-adjoint solutions of the CARE, and if $A_{Q_{1}}$ and $A_{Q_{2}}$ are defined similarly as $A_{Q}$ above, then

$$
Q_{1}-Q_{2}=A_{Q_{2}}^{*}\left(Q_{1}-Q_{2}\right) A_{Q_{1}} .
$$

With induction it follows that for all $n \in \mathbb{N}$ we have

$$
Q_{1}-Q_{2}=A_{Q_{2}}^{* n}\left(Q_{1}-Q_{2}\right) A_{Q_{1}}^{n} .
$$

Using these facts we now prove the statement. Since $(A, B, C, D)$ is input stabilizable, there exists a nonnegative self-adjoint solution $P$ of the FARE (9). Since $A_{Q}$ is assumed to be strongly stable and (10) shows that $A_{P}$ is similar to $A_{Q}$, we have that $A_{P}$ is strongly stable. Now let $\tilde{Q}$ be an arbitrary nonnegative self-adjoint solution of the CARE. According to (10), $A_{\tilde{Q}}$ is similar to the strongly stable operator $A_{P}$ and hence is strongly stable. Since $A_{\tilde{Q}}$ is strongly stable there exists for every $x \in X$ a real number $c_{x}$ such that for every $n \in \mathbb{N}$ we have $\left\|A_{\tilde{Q}}^{n} x\right\| \leq c_{x}$. By the uniform boundedness theorem this implies that there exists a real number $c$ such that for every $n \in \mathbb{N}$ we have $\left\|A_{\tilde{Q}}^{n}\right\| \leq c$. Using (12) with $Q_{1}=Q$ and $Q_{2}=\tilde{Q}$ we have for all $x \in X$ and $n \in \mathbb{N}$

$\|(Q-\tilde{Q}) x\|=\left\|A_{\tilde{Q}}^{* n}(Q-\tilde{Q}) A_{Q}^{n} x\right\| \leq\left\|A_{\tilde{Q}}^{* n}\right\|\|Q-\tilde{Q}\|\left\|A_{Q}^{n} x\right\| \leq c\|Q-\tilde{Q}\|\left\|A_{Q}^{n} x\right\|$.

Since $A_{Q}$ is strongly stable, the right-hand side converges to zero as $n \rightarrow \infty$. This implies that the left-hand side is zero and so $\tilde{Q}=Q$.

The input-output map $\mathcal{D}$ of a discrete-time system $(A, B, C, D)$ is defined for finitely nonzero $U$-valued sequences $u$ by

$$
(\mathcal{D} u)_{k}:=\sum_{i=0}^{\infty} C A^{i} B u_{k-i-1}+D u_{k} .
$$

The discrete-time system $(A, B, C, D)$ is said to be input-output stable if $\mathcal{D}$ extends to a bounded map from $l^{2}(\mathbb{Z} ; U)$ to $l^{2}(\mathbb{Z} ; Y)$.

We define the transfer function $G$ of a system $(A, B, C, D)$ by

$$
G(z)=D+\sum_{i=0}^{\infty} C A^{i} B z^{i+1}
$$


for those $z$ for which the sum converges absolutely. Note that it converges absolutely for $|z|<1 / r(A)(r(A)$ denotes the spectral radius of $A)$ and it is equal to $D+C z(I-z A)^{-1} B$ for those $z$. It is obvious that the transfer function of a system can be constructed from the input-output map and vice versa; in this sense transfer functions and input-output maps are equivalent notions. Given a transfer function $G$ we call any system $(A, B, C, D)$ such that (14) holds a realization of the transfer function. We note that the functions that appear as transfer functions of discrete-time infinite-dimensional systems are exactly the operator-valued functions that are analytic on some disc centered at the origin.

The (time-domain) Hankel operator $\Gamma$ of a system is defined as $\Gamma:=\mathcal{C B}$, where $\mathcal{C}$ and $\mathcal{B}$ are the observability and controllability maps of the system, respectively. It is easily seen that the Hankel operator does not depend on the particular realization but only on the input-output map.

\section{Lyapunov-balanced realizations}

In this section we review some results from Young [15], [16] and Ober and Wu [10] and translate them in terms more suitable for our purposes. The following result on the existence of Lyapunov-balanced realizations was proved by Young [15], [16]. We recall that an input and output stable system is called Lyapunov balanced if its controllability and observability gramians are equal (again, we do not require them to be diagonal).

Lemma 4.1. Every transfer function that has a bounded Hankel operator has an approximately controllable and observable Lyapunov-balanced realization. Moreover, approximately controllable and observable Lyapunov-balanced realizations are unique up to a unitary transformation.

The next corollary gives an alternative condition for the existence of Lyapunovbalanced realizations.

Corollary 4.2. A transfer function has a Lyapunov-balanced realization if and only if it has a realization that is both input and output stable.

Proof. Since a Lyapunov-balanced realization is input and output stable, one implication is immediate. If the transfer function has a realization such that both

its controllability map $\mathcal{B}$ and observability map $\mathcal{C}$ are bounded, then its Hankel operator $\Gamma=\mathcal{C B}$ is bounded and Lemma 4.1 shows that it has a Lyapunovbalanced realization.

The following result was proved by Ober and $\mathrm{Wu}[10]$.

Lemma 4.3. Let $(A, B, C, D)$ be Lyapunov-balanced and approximately controllable and approximately observable. Then $A$ and $A^{*}$ are strongly stable.

Combining Lemmas 4.3, 3.2, and 3.5 we have the following corollary. 
Corollary 4.4. The gramian of an approximately controllable and approximately observable Lyapunov-balanced realization is the unique nonnegative selfadjoint solution of both the control and the observation Lyapunov equation.

\section{$5 \quad$ Normalized factorizations}

In this section we generalize a result of Meyer and Franklin [6] on the connection between normalized factorizations and linear quadratic regulator theory to the infinite-dimensional case. This result will allow us to relate LQG-balanced realizations to Lyapunov-balanced realizations of a normalized factorization of the given transfer function.

Given an output stabilizable discrete-time system $(A, B, C, D)$ with optimal cost operator $Q$ we form the optimal closed-loop system

$$
\check{A}:=A+B F, \quad \check{B}:=B W^{-1 / 2}, \quad \check{C}:=[F ; C+D F], \quad \check{D}:=[I ; D] W^{-1 / 2},
$$

where

$$
W:=S+B^{*} Q B, \quad S:=I+D^{*} D, \quad F:=-W^{-1}\left(D^{*} C+B^{*} Q A\right) .
$$

We first remark that the $F$ above is the optimal state feedback operator for the LQR problem. We obtain the optimal closed-loop system from the system $(A, B, C, D)$ by choosing $u=F x+W^{-1 / 2} \check{u}$ and considering $\check{u}$ as the input of this new system and $[u ; y]$ as the output. This amounts to closing the loop by the optimal state feedback operator, considering the input and output of the plant as the new output and prefiltering the new input.

Our first result in this section states that the optimal cost operator of the plant equals the observability gramian of the optimal closed-loop system.

Lemma 5.1. Let $(A, B, C, D)$ be an output stabilizable discrete-time system. Denote its optimal cost operator by $Q$ and define its optimal closed-loop system by (15). Denote the observability gramian of the optimal closed-loop system by $L_{C}$. Then $Q=L_{C}$.

Proof. From the discussion above it is obvious that if $\check{u}=0$, then the output of the optimal closed-loop system is $\left[u^{\mathrm{min}} ; y^{\mathrm{min}}\right]$, the optimal input and output of the plant. From this it follows that

$$
\left\langle L_{C} x_{0}, x_{0}\right\rangle=\left\langle\mathcal{C} x_{0}, \mathcal{C} x_{0}\right\rangle=\left\|\left[\begin{array}{l}
u^{\min } \\
y^{\min }
\end{array}\right]\right\|^{2}=J\left(x_{0}, u^{\min }\right)=\left\langle Q x_{0}, x_{0}\right\rangle .
$$

Since this holds for all $x_{0}$ in the state space we have $Q=L_{C}$.

The next lemma shows that the observability gramian of the optimal closedloop system satisfies two additional equations.

Lemma 5.2. Let $(A, B, C, D)$ be an output stabilizable discrete-time system. Denote its optimal cost operator by $Q$ and define its optimal closed-loop system 
$(\check{A}, \check{B}, \check{C}, \check{D})$ by (15). Denote the observability gramian of the optimal closed-loop system by $L_{C}$. Then

$$
\begin{aligned}
\check{B}^{*} L_{C} \check{B}+\check{D}^{*} \check{D} & =I, \\
\check{B}^{*} L_{C} \check{A}+\check{D}^{*} \check{C} & =0 .
\end{aligned}
$$

Proof. The equations (16) and (17) are readily verified using (15) and the fact that $Q=L_{C}$ from Lemma 5.1.

An input-output map $\mathcal{D}$ is called inner if it maps $l^{2}(\mathbb{Z} ; U)$ into $l^{2}(\mathbb{Z} ; Y)$ and satisfies $\mathcal{D}^{*} \mathcal{D}=I$. In the next lemma we give necessary and sufficient conditions on a realization for the input-output map to be inner.

Lemma 5.3. Let $(A, B, C, D)$ be an output stable realization of the input-output map $\mathcal{D}$. Denote the observability gramian of this system by $L_{C}$. If

$$
B^{*} L_{C} B+D^{*} D=I
$$

and

$$
B^{*} L_{C} A+D^{*} C=0,
$$

then $\mathcal{D}$ is inner. If the system $(A, B, C, D)$ is approximately controllable, then these conditions are also necessary.

Proof. We take $u_{k}$ equal to $u$ at the $k$ th position and zero elsewhere and compute

$$
\left(\mathcal{D} u_{k}\right)_{m}= \begin{cases}0, & m<k, \\ D u, & m=k, \\ C A^{m-k-1} B u, & m>k .\end{cases}
$$

We define $v_{i}$ similar to $u_{k}$ above and compute for $k>i$

$$
\left\langle\left(\mathcal{D} u_{k}\right)_{n},\left(\mathcal{D} v_{i}\right)_{n}\right\rangle= \begin{cases}0, & n<k, \\ \left\langle D u, C A^{k-i-1} B v\right\rangle, & n=k, \\ \left\langle C A^{n-k-1} B u, C A^{n-i-1} B v\right\rangle, & n>k\end{cases}
$$

We then compute

$$
\begin{aligned}
\left\langle\mathcal{D} u_{k}, \mathcal{D} v_{i}\right\rangle & =\sum_{n=-\infty}^{\infty}\left\langle\left(\mathcal{D} u_{k}\right)_{n},\left(\mathcal{D} v_{i}\right)_{n}\right\rangle \\
& =\left\langle D u, C A^{k-i-1} B v\right\rangle+\sum_{n=k+1}^{\infty}\left\langle C A^{n-k-1} B u, C A^{n-i-1} B v\right\rangle \\
& =\left\langle u, D^{*} C A^{k-i-1} B v\right\rangle+\sum_{n=k+1}^{\infty}\left\langle u, B^{*} A^{* n-k-1} C^{*} C A^{n-k-1} A A^{k-i-1} B v\right\rangle \\
& =\left\langle u,\left(D^{*} C+B^{*} L_{C} A\right) A^{k-i-1} B v\right\rangle .
\end{aligned}
$$


By the assumptions we thus have $\left\langle\mathcal{D} u_{k}, \mathcal{D} v_{i}\right\rangle=0$ for $k>i$. Then obviously $\left\langle\mathcal{D} u_{k}, \mathcal{D} v_{i}\right\rangle$

$=0$ for $k \neq i$.

For $k=i$ we have

$$
\begin{aligned}
\left\langle\mathcal{D} u_{k}, \mathcal{D} v_{i}\right\rangle & =\sum_{n=-\infty}^{\infty}\left\langle\left(\mathcal{D} u_{k}\right)_{n},\left(\mathcal{D} v_{i}\right)_{n}\right\rangle \\
& =\langle D u, D v\rangle+\sum_{n=k+1}^{\infty}\left\langle C A^{n-k-1} B u, C A^{n-k-1} B v\right\rangle \\
& =\left\langle D^{*} D u, v\right\rangle+\sum_{j=0}^{\infty}\left\langle B^{*} A^{* j} C^{*} C A^{j} B u, v\right\rangle=\left\langle\left(D^{*} D+B^{*} L_{C} B\right) u, v\right\rangle .
\end{aligned}
$$

By the assumptions we thus have $\left\langle\mathcal{D} u_{k}, \mathcal{D} v_{i}\right\rangle=\langle u, v\rangle$ for $k=i$.

Let $u$ and $v$ be finitely nonzero sequences. Then

$$
u=\sum_{k=-n}^{n} u^{k} e_{k}, \quad v=\sum_{i=-n}^{n} v^{i} e_{i},
$$

where $u^{k}, v^{i} \in U$ and $e_{j}$ is the element of $l^{2}(\mathbb{Z} ; U)$ with a 1 at the $j$ th position and zeros elsewhere. Then

$$
\begin{aligned}
\langle\mathcal{D} u, \mathcal{D} v\rangle & =\sum_{k=-n}^{n} \sum_{i=-n}^{n}\left\langle\mathcal{D}\left(u^{k} e_{k}\right), \mathcal{D}\left(v^{i} e_{i}\right)\right\rangle=\sum_{j=-n}^{n}\left\langle\mathcal{D}\left(u^{j} e_{j}\right), \mathcal{D}\left(v^{j} e_{j}\right)\right\rangle \\
& =\sum_{j=-n}^{n}\left\langle u^{j}, v^{j}\right\rangle=\langle u, v\rangle .
\end{aligned}
$$

From the above we have for every finitely nonzero sequence $u$ that $\|\mathcal{D} u\|=\|u\|$. Since the set of finitely nonzero sequences is dense in $l^{2}(\mathbb{Z} ; U)$ this implies that $\mathcal{D}$ has a continuous extension to a map from $l^{2}(\mathbb{Z} ; U)$ to $l^{2}(\mathbb{Z} ; Y)$, and so $\mathcal{D}$ is stable. Further, since this extension satisfies $\langle\mathcal{D} u, \mathcal{D} v\rangle=\langle u, v\rangle$, we must have $\mathcal{D}^{*} \mathcal{D}=I$, and so $\mathcal{D}$ is inner.

Suppose that $\mathcal{D}$ is inner and the realization is approximately controllable. Since $\mathcal{D}$ is inner we have for all $i<0$ that $\left\langle\mathcal{D} u_{0}, \mathcal{D} v_{i}\right\rangle=\left\langle u_{0}, v_{i}\right\rangle=0$, where $u_{0}$ and $v_{i}$ are defined as above. From the above we see that this implies that $\left\langle u,\left(D^{*} C+B^{*} L_{C} A\right) A^{-i-1} B v\right\rangle=0$. Since this holds for all $u \in U$ we must have $\left(D^{*} C+B^{*} L_{C} A\right) A^{-i-1} B v=0$ for all $v \in U$ and $i<0$. This implies that for finitely nonzero $U$-valued sequences $z$ we have $\left(D^{*} C+B^{*} L_{C} A\right) \mathcal{B} z=0$. Since the system is approximately controllable, the set of elements of the form $\mathcal{B} z$ is dense in the state space, and so $D^{*} C+B^{*} L_{C} A=0$ on a dense set and by continuity on the whole state space.

Since $\mathcal{D}$ is inner we have $\left\langle\mathcal{D} u_{0}, \mathcal{D} v_{0}\right\rangle=\left\langle u_{0}, v_{0}\right\rangle$, where $u_{0}$ and $v_{0}$ are as above. This implies that $\left\langle\left(D^{*} D+B^{*} L_{C} B\right) u, v\right\rangle=\langle u, v\rangle$ for all $u, v \in U$ and hence $D^{*} D+B^{*} L_{C} B=I$. 
Combining Lemmas 5.2 and 5.3 we have the following.

Corollary 5.4. Let $(A, B, C, D)$ be an output stabilizable discrete-time system. Then the input-output map of its optimal closed-loop system is inner.

We can recover the system $(A, B, C, D)$ from its optimal closed-loop system as follows.

Lemma 5.5. Let $(A, B, C, D)$ be an output stabilizable discrete-time system. Let $(\check{A}, \check{B}, \check{C}, \check{D})$ be its optimal closed-loop system defined by (15). Partition $\check{C}$ and $\check{D}$ in the obvious way as $\check{C}=\left[\check{C}_{1} ; \check{C}_{2}\right]$ and $\check{D}=\left[\check{D}_{1} ; \check{D}_{2}\right]$. Then $\check{D}_{1}$ is boundedly invertible and

$$
A:=\check{A}-\check{B} \check{D}_{1}^{-1} \check{C}_{1}, \quad B:=\check{B} \check{D}_{1}^{-1}, \quad C:=\check{C}_{2}-\check{D}_{2} \check{D}_{1}^{-1} \check{C}_{1}, \quad D:=\check{D}_{2} \check{D}_{1}^{-1} .
$$

Proof. That $\check{D}_{1}$ is boundedly invertible is obvious from its definition. The identities (18) follow from simple algebraic manipulations.

To relate the input-output maps of the plant and its optimal closed-loop system we first study the series interconnection of two systems.

Consider two systems $\left(A_{1}, B_{1}, C_{1}, D_{1}\right)$ and $\left(A_{2}, B_{2}, C_{2}, D_{2}\right)$ such that the output space of the first system and the input space of the second system are equal. Define the series interconnection of these two systems as the system we obtain by choosing the input of the second system equal to the output of the first system. Obviously, the input-output map of the series interconnection is $\mathcal{D}_{2} \mathcal{D}_{1}$, the composition of the input-output maps of the first and second systems. A realization of this series interconnection is the following:

$$
A=\left[\begin{array}{cc}
A_{1} & 0 \\
B_{2} C_{1} & A_{2}
\end{array}\right], \quad B=\left[\begin{array}{c}
B_{1} \\
B_{2} D_{1}
\end{array}\right], \quad C=\left[\begin{array}{cc}
D_{2} C_{1}, & C_{2}
\end{array}\right], \quad D=D_{2} D_{1} .
$$

If we apply the invertible state space transformation

$$
\left[\begin{array}{ll}
I & 0 \\
I & I
\end{array}\right]
$$

to this realization we obtain another realization of the series interconnection, namely,

$$
\begin{gathered}
A_{s}=\left[\begin{array}{cc}
A_{1} & 0 \\
A_{2}+B_{2} C_{1}-A_{1} & A_{2}
\end{array}\right], \quad B_{s}=\left[\begin{array}{c}
B_{1} \\
B_{2} D_{1}-B_{1}
\end{array}\right], \\
C_{s}=\left[\begin{array}{ll}
D_{2} C_{1}+C_{2}, & C_{2}
\end{array}\right], \quad D_{s}=D_{2} D_{1} .
\end{gathered}
$$

We first use the series interconnection to obtain a result about the invertibility of input-output maps.

Lemma 5.6. Let $(A, B, C, D)$ be a system with input-output map $\mathcal{D}$. If $D$ is boundedly invertible, then the input-output map $\tilde{\mathcal{D}}$ of the system

$$
\left(A-B D^{-1} C, B D^{-1},-D^{-1} C, D^{-1}\right)
$$

satisfies $\tilde{\mathcal{D}} \mathcal{D}=I=\mathcal{D} \tilde{\mathcal{D}}$. Thus in this case the input-output map $\mathcal{D}$ has an inverse. 
Proof. Using (19) we see that the series interconnection of the two given systems has a realization

$$
A_{s}=\left[\begin{array}{cc}
A & 0 \\
0 & A-B D^{-1} C
\end{array}\right], \quad B_{s}=\left[\begin{array}{c}
B \\
0
\end{array}\right], \quad C_{s}=\left[\begin{array}{cc}
0, & -D^{-1} C
\end{array}\right], \quad D_{s}=I .
$$

From this we see that $C_{s} A_{s}^{k} B_{s}=0$ for all $k \geq 0$ and so the input-output map of the series interconnection is the identity. This implies that $\tilde{\mathcal{D}} \mathcal{D}=I$. The other equality mentioned follows from interconnecting the systems in the opposite order.

We now state the relation between the input-output map of a plant and its optimal closed-loop system.

Lemma 5.7. Let $\left(\check{A}, \check{B},\left[\check{C}_{1} ; \check{C}_{2}\right],\left[\check{D}_{1} ; \check{D}_{2}\right]\right)$ be a system such that $\check{D}_{1}$ is boundedly invertible. Denote its input-output map by $[\mathcal{M} ; \mathcal{N}]$. Define the system $(A, B, C, D)$ by $(18)$ and denote its input-output map by $\mathcal{D}$. Then $\mathcal{D}=\mathcal{N M}^{-1}$.

Proof. The realization $\left(\check{A}, \check{B},\left[\check{C}_{1} ; \check{C}_{2}\right],\left[\check{D}_{1} ; \check{D}_{2}\right]\right)$ of $[\mathcal{M} ; \mathcal{N}]$ gives us (by Lemma 5.6) the following realization $\left(A_{1}, B_{1}, C_{1}, D_{1}\right)$ of $\mathcal{M}^{-1}$ :

$$
A_{1}=\check{A}-\check{B} \check{D}_{1}^{-1} \check{C}_{1}, \quad B_{1}=\check{B} \check{D}_{1}^{-1}, \quad C_{1}=-\check{D}_{1}^{-1} \check{C}_{1}, \quad D_{1}=\check{D}_{1}^{-1} .
$$

It also gives us the realization $\left(A_{2}, B_{2}, C_{2}, D_{2}\right)$ of $\mathcal{N}$ :

$$
A_{2}=\check{A}, \quad B_{2}=\check{B}, \quad C_{2}=\check{C}_{2}, \quad D_{2}=\check{D}_{2} .
$$

Using (19) we obtain the following realization of the series interconnection which has input-output map $\mathcal{N} \mathcal{M}^{-1}$ :

$$
\begin{gathered}
A_{s}=\left[\begin{array}{cc}
\check{A}-\check{B} \check{D}_{1}^{-1} \check{C}_{1} & 0 \\
0 & \check{A}
\end{array}\right]=\left[\begin{array}{cc}
A & 0 \\
0 & \check{A}
\end{array}\right], \quad B_{s}=\left[\begin{array}{c}
\check{B}_{\check{D}}^{-1} \\
0
\end{array}\right]=\left[\begin{array}{c}
B \\
0
\end{array}\right], \\
C_{s}=\left[\check{C}_{2}-\check{D}_{2} \check{D}_{1}^{-1} \check{C}_{1}, \quad \check{C}_{2}\right]=\left[\begin{array}{ll}
C, & \check{C}_{2}
\end{array}\right], \quad D_{s}=\check{D}_{2} \check{D}_{1}^{-1}=D,
\end{gathered}
$$

where we have used (18). It follows that $D_{s}=D$ and $C_{s} A_{s}^{k} B_{s}=C A^{k} B$ for all $k \geq 0$. This implies that $\mathcal{N} \mathcal{M}^{-1}=\mathcal{D}$.

We call an input-output map $[\mathcal{M} ; \mathcal{N}]$ a right factor of the input-output map $\mathcal{D}$ if $\mathcal{M}^{-1}$ is the input-output map of a system, $\mathcal{D}=\mathcal{N} \mathcal{M}^{-1}$ and $\mathcal{M}$ and $\mathcal{N}$ are stable. We call the factor normalized if $[\mathcal{M} ; \mathcal{N}]$ is inner. From Lemmas 5.5 and 5.7 and Corollary 5.4 we have the following.

Corollary 5.8. Let $(A, B, C, D)$ be an output stabilizable discrete-time system. Then the input-output map of its optimal closed-loop system is a normalized right factor of the input-output map of the plant.

We next state a result about the uniqueness of a normalized right factor. We remark that we can interpret an operator $V$ in $\mathcal{L}(U)$ as a map $\mathcal{V}$ from $l^{2}(\mathbb{Z} ; U)$ into itself by $(\mathcal{V} u)_{k}=V u_{k}$. 
Lemma 5.9. If the input-output map of a system has a normalized right factor $[\mathcal{M} ; \mathcal{N}]$, then all normalized right factors of this input-output map are $\{[\mathcal{M V} ; \mathcal{N} V]:$ $V \in \mathcal{L}(U)$ unitary $\}$.

Proof. Let $[\mathcal{M} ; \mathcal{N}]$ be an arbitrary normalized right factor of $\mathcal{D}$. Since $[\mathcal{M} ; \mathcal{N}]$ is normalized we have

$$
\mathcal{M}^{*} \mathcal{M}+\mathcal{N}^{*} \mathcal{N}=I .
$$

Multiplying this equality with $\mathcal{M}^{-*}$ from the left and $\mathcal{M}^{-1}$ from the right we obtain

$$
I+\mathcal{D}^{*} \mathcal{D}=\mathcal{M}^{-*} \mathcal{M}^{-1} .
$$

Since the left-hand side of this equation does not depend on the particular factor, we have for two normalized factors $\left[\mathcal{M}_{1} ; \mathcal{N}_{1}\right]$ and $\left[\mathcal{M}_{2} ; \mathcal{N}_{2}\right]$ of $\mathcal{D}$ that $\mathcal{M}_{1}^{-*} \mathcal{M}_{1}^{-1}=\mathcal{M}_{2}^{-*} \mathcal{M}_{2}^{-1}$, which implies

$$
\mathcal{M}_{2}^{*} \mathcal{M}_{1}^{-*}=\mathcal{M}_{2}^{-1} \mathcal{M}_{1}
$$

The operator on the right-hand side of (20) is the input-output map of some system (namely, the series interconnection of the systems corresponding to $\mathcal{M}_{1}$ and $\left.\mathcal{M}_{2}^{-1}\right)$. Define $u_{k}$ to be the sequence equal to $u$ at the $k$ th position and zero elsewhere. Then $\mathcal{M}_{2}^{-1} \mathcal{M}_{1} u_{k}$ is equal to zero at the positions $i$ with $i<k$. The operator on the left-hand side of (20) is the adjoint of the input-output map of some system (namely, the series interconnection of the systems corresponding to $\mathcal{M}_{2}$ and $\left.\mathcal{M}_{1}^{-1}\right)$. This implies that $\mathcal{M}_{2}^{*} \mathcal{M}_{1}^{-*} u_{k}$ is zero at the positions $i$ with $i>k$.

Since $\mathcal{M}_{2}^{*} \mathcal{M}_{1}^{-*}=\mathcal{M}_{2}^{-1} \mathcal{M}_{1}$ we must have that $\mathcal{M}_{2}^{-1} \mathcal{M}_{1} u_{k}$ is equal to zero at all positions except possibly the $k$ th one. Thus $\mathcal{M}_{2}^{-1} \mathcal{M}_{1}$ is a constant operator $V$, and so $\mathcal{M}_{1}=\mathcal{M}_{2} V$.

We have $\mathcal{N}_{1}=\mathcal{D} \mathcal{M}_{1}=\mathcal{D} \mathcal{M}_{2} V=\mathcal{N}_{2} V$.

It remains to be proved that $V$ is unitary. We have that $V=\mathcal{M}_{2}^{-1} \mathcal{M}_{1}$ and so $V^{*}=\mathcal{M}_{1}^{*} \mathcal{M}_{2}^{-*}=\mathcal{M}_{1}^{-1} \mathcal{M}_{2}=V^{-1}$ by (20). This proves that $V$ is unitary.

In the finite-dimensional case the transfer function of the optimal closedloop system is known to be a normalized coprime factorization. In the infinitedimensional case this is an open problem.

\section{Some algebraic relations}

In section 5 we proved that the optimal cost operator equals the observability gramian of the optimal closed-loop system. This can also be interpreted as follows. The smallest nonnegative self-adjoint solution of the control algebraic Riccati equation equals the smallest nonnegative self-adjoint solution of the observation Lyapunov equation of a certain closed-loop system. In this section we show that a similar result holds for all nonnegative self-adjoint solutions of the control algebraic Riccati equation. We also study the relation between nonnegative self-adjoint solutions of the filter algebraic Riccati equation of the 
plant and nonnegative self-adjoint solutions of the control Lyapunov equation of the closed-loop system.

The CARE closed-loop system $(\check{A}, \check{B}, \check{C}, \check{D})$ associated with an output stabilizable discrete-time system $(A, B, C, D)$ and a nonnegative self-adjoint solution $Q$ of the CARE (5) is defined by (15). For the special case that $Q$ is the smallest nonnegative self-adjoint solution of the CARE (5) the CARE closed-loop system is equal to the optimal closed-loop system defined earlier.

Lemma 5.5 holds in this more general case, which is obvious from its proof.

Lemma 6.1. Let $(A, B, C, D)$ be a discrete-time system such that its $C A R E$ (5) has a nonnegative self-adjoint solution $Q$. Let $(\check{A}, \check{B}, \check{C}, \check{D})$ be its $C A R E$ closed-loop system defined by (15). Partition $\check{C}$ and $\check{D}$ in the obvious way as $\check{C}=\left[\check{C}_{1} ; \check{C}_{2}\right]$ and $\check{D}=\left[\check{D}_{1} ; \check{D}_{2}\right]$. Then $\check{D}_{1}$ is boundedly invertible and (18) holds.

This lemma implies that the input-output map of the CARE closed-loop system is a factor of the input-output map of the plant by Lemma 5.7. If $Q$ is not the smallest nonnegative self-adjoint solution of the CARE, the factorization need not be normalized (see, e.g., [11, Example 3.1.2]).

We now show that if a system is approximately controllable or observable, then its CARE closed-loop system is too.

Lemma 6.2. Let $(A, B, C, D)$ be an output stabilizable discrete-time system and let $Q$ be a nonnegative self-adjoint solution of its $C A R E(5)$. If $(A, B, C, D)$ is approximately controllable, then its CARE closed-loop system is too. If $(A, B, C, D)$ is approximately observable, then its CARE closed-loop system is too.

Proof. As is well known, a system $(\check{A}, \check{B}, \check{C}, \check{D})$ is approximately controllable (observable) iff $(\check{A}+\check{B} K \check{C}, \check{B}, \check{C}, \check{D})$ is approximately controllable (observable). With $K=-W^{1 / 2}[I, 0]$ we see that the CARE closed-loop system is approximately controllable (observable) iff $(A, \breve{B}, \check{C}, \check{D})$ is approximately controllable (observable). Obviously $(A, \check{B})=\left(A, B W^{-1 / 2}\right)$ is approximately controllable iff $(A, B)$ is approximately controllable and $(A, \breve{C})=(A,[F ; C+D F])$ is approximately observable if $(A, C)$ is (but not only if).

In the next four lemmas we prove a correspondence between the Riccati equations of a system and the Lyapunov equations of its CARE closed-loop system. Because this requires some extensive algebraic manipulations we have relegated some of the proofs to the appendix.

Lemma 6.3. Let $(A, B, C, D)$ be an output stabilizable discrete-time system and let $Q$ be a nonnegative self-adjoint solution of its CARE (5). Let $(\check{A}, \check{B}, \check{C}, \check{D})$ be its CARE closed-loop system defined by (15). Then

$$
\begin{aligned}
& \check{B}^{*} Q \check{B}+\check{D}^{*} \check{D}=I, \\
& \check{B}^{*} Q \check{A}+\check{D}^{*} \check{C}=0,
\end{aligned}
$$

and $Q$ is a solution of the observation Lyapunov equation of $(\check{A}, \check{B}, \check{C}, \check{D})$ :

$$
\check{A}^{*} Q \check{A}-Q+\check{C}^{*} \check{C}=0 .
$$


Proof. The equations (21) and (22) are readily verified using (15). Equation (23) is more complicated and the proof is given in the appendix.

Lemma 6.4. Let $\left(\check{A}, \check{B},\left[\check{C}_{1} ; \check{C}_{2}\right],\left[\check{D}_{1}, \check{D}_{2}\right]\right)$ be a discrete-time system such that $\check{D}_{1}$ is boundedly invertible and define the discrete-time system $(A, B, C, D)$ by (18). If the nonnegative self-adjoint operator $Q$ satisfies (22) and (23), then $Q$ is a solution of the CARE (5) of $(A, B, C, D)$.

Proof. See the appendix.

Lemma 6.5. Let $(A, B, C, D)$ be an input and output stabilizable discrete-time system and let $Q$ be a nonnegative self-adjoint solution of its $C A R E(5)$ and $P$ be a nonnegative self-adjoint solution of its FARE (9). Let $(\check{A}, \check{B}, \check{C}, \check{D})$ be its $C A R E$ closed-loop system defined by (15). Define $L:=(I+P Q)^{-1} P$. Then $L$ is a solution of the control Lyapunov equation of $(\check{A}, \check{B}, \check{C}, \check{D})$ :

$$
\check{A} L \check{A}^{*}-L+\check{B} \check{B}^{*}=0 .
$$

Proof. See the appendix for the proof.

Lemma 6.6. Let $\left(\check{A}, \breve{B},\left[\check{C}_{1} ; \check{C}_{2}\right],\left[\check{D}_{1}, \check{D}_{2}\right]\right)$ be a discrete-time system such that $\check{D}_{1}$ is boundedly invertible and define the discrete-time system $(A, B, C, D)$ by (18). If the nonnegative self-adjoint operators $Q$ and $L$ satisfy (21), (22), (23), and (24) and $I-Q L$ is boundedly invertible, then $L(I-Q L)^{-1}$ is a solution of the FARE (9) of $(A, B, C, D)$.

Proof. See the appendix for the proof.

If $Q$ and $P$ are the smallest nonnegative self-adjoint solutions of their respective Riccati equations, then the operator $L:=(I+P Q)^{-1} P$ defined in Lemma 6.5 is actually the smallest nonnegative self-adjoint solution of the Lyapunov equation (24). To prove this we first prove the following lemma. We note that the Hankel norm of an input-output map is the norm of the associated Hankel operator.

Lemma 6.7. Let $(A, B, C, D)$ be an input and output stabilizable discrete-time system. Then the Hankel norm of the input-output map of the optimal closedloop system (15) is strictly smaller than one.

Proof. Denote the optimal cost operators by $P$ and $Q$, the gramians of the optimal closed-loop system by $L_{B}$ and $L_{C}$, and the Hankel operator of the optimal closed-loop system by $\Gamma$. From Lemma 5.1 it follows that $Q=L_{C}$. From Lemma 6.5 we know that $(I+P Q)^{-1} P$ is a solution of the control Lyapunov equation and hence by Lemma 3.4 we have $L_{B} \leq(I+P Q)^{-1} P$. This implies that $L_{C}^{1 / 2} L_{B} L_{C}^{1 / 2} \leq Q^{1 / 2}(I+P Q)^{-1} P Q^{1 / 2}=\left(I+Q^{1 / 2} P Q^{1 / 2}\right)^{-1} Q^{1 / 2} P Q^{1 / 2}$. Now $\|\Gamma\|=\left\|\Gamma \Gamma^{*}\right\|=r\left(\Gamma \Gamma^{*}\right)=r\left(\mathcal{C B B}^{*} \mathcal{C}^{*}\right)=r\left(\mathcal{C}^{*} \mathcal{C B B}^{*}\right)=r\left(L_{C} L_{B}\right)=$ $r\left(L_{C}^{1 / 2} L_{B} L_{C}^{1 / 2}\right) \leq r\left(\left(I+Q^{1 / 2} P Q^{1 / 2}\right)^{-1} Q^{1 / 2} P Q^{1 / 2}\right)$. Next we show that if $X$ is a nonnegative self-adjoint operator, then $(I+X)^{-1} X<I$. Since $X<I+X$ we have $(I+X)^{-1} X=(I+X)^{-1 / 2} X(I+X)^{-1 / 2}<(I+X)^{-1 / 2}(I+X)(I+$ 
$X)^{-1 / 2}=I$. Finally, we apply this last result with $X:=Q^{1 / 2} P Q^{1 / 2}$ to obtain $\|\Gamma\| \leq r\left((I+X)^{-1} X\right)<1$.

Lemma 6.7 has the following corollary.

Corollary 6.8. Let $(A, B, C, D)$ be an input and output stabilizable discretetime system. Let $L_{B}$ and $L_{C}$ denote the gramians of an input and output stable realization of the input-output map of the optimal closed-loop system (15). Then $r\left(L_{B} L_{C}\right)<1$.

We now show for an approximately observable system that if $Q$ and $P$ are the smallest nonnegative self-adjoint solutions of their respective Riccati equations then the operator $L:=(I+P Q)^{-1} P$ defined in Lemma 6.5 is actually the smallest nonnegative self-adjoint solution of the Lyapunov equation (24).

Lemma 6.9. Let $(A, B, C, D)$ be an approximately observable input and output stabilizable discrete-time system. Let $Q$ and $P$ denote the optimal cost operators of the system and its dual, respectively. Define $L:=(I+P Q)^{-1} P$. Then $L$ is the controllability gramian of the optimal closed-loop system (15).

Proof. We have by Lemma 5.1 that $Q=L_{C}$, the observability gramian of the optimal closed-loop system, and by Lemma 6.5 that $L=(I+P Q)^{-1} P$ is a solution of the control Lyapunov equation of the optimal closed-loop system. Since the control Lyapunov equation of the optimal closed-loop system has a nonnegative self-adjoint solution, the optimal closed-loop system is input stable and has a controllability gramian $L_{B}$ which satisfies $L_{B} \leq L$. From Lemma 6.8 we know that $I-L_{C} L_{B}$ is boundedly invertible and hence by Lemma 6.6 we have that $\tilde{P}:=L_{B}\left(I-L_{C} L_{B}\right)^{-1}$ is a solution of the FARE (9) of the system $(A, B, C, D)$. We thus have $P \leq \tilde{P}$. Note that approximate observability ensures that $Q=L_{C}>0$. Since $Q$ is positive and thus has dense range we have $\tilde{P} \geq P$ iff $Q^{1 / 2} \tilde{P} Q^{1 / 2} \geq Q^{1 / 2} P Q^{1 / 2}$. This is true iff

$$
\begin{aligned}
I-Q^{1 / 2}(I+\tilde{P} Q)^{-1} \tilde{P} Q^{1 / 2} & =\left(I+Q^{1 / 2} \tilde{P} Q^{1 / 2}\right)^{-1} \\
\leq & \left(I+Q^{1 / 2} P Q^{1 / 2}\right)^{-1}=I-Q^{1 / 2}(I+P Q)^{-1} P Q^{1 / 2} .
\end{aligned}
$$

This is true iff $Q^{1 / 2}(I+\tilde{P} Q)^{-1} \tilde{P} Q^{1 / 2} \geq Q^{1 / 2}(I+P Q)^{-1} P Q^{1 / 2}$ and again using that $Q$ has dense range this is true iff $(I+\tilde{P} Q)^{-1} \tilde{P} \geq(I+P Q)^{-1} P$. We conclude that $L=(I+P Q)^{-1} P \leq(I+\tilde{P} Q)^{-1} \tilde{P}=L_{B}$. Since we already had $L_{B} \leq L$, we must have $L=L_{B}$.

\section{LQG-characteristic values}

In this section we show that the spectrum of the product of the optimal cost operators of a system and its dual does not depend on the realization but only on the input-output map. This generalizes the result from finite-dimensional theory that the eigenvalues of $P Q$ are similarity invariants. We define the LQGcharacteristic values of an input and output stabilizable discrete-time system to be the square roots of the spectral values of the product of the optimal cost operators $P$ and $Q$. We first prove the following lemma on the spectrum of $P Q$. 
Lemma 7.1. Let $(A, B, C, D)$ be an approximately observable input and output stabilizable discrete-time system. Let $Q$ and $P$ denote the optimal cost operators of the system and its dual, respectively, and let $L_{B}$ and $L_{C}$ denote the gramians of the optimal closed-loop system (15). Then $\lambda \in \sigma(P Q)$ iff $\lambda /(1+\lambda) \in \sigma\left(L_{B} L_{C}\right)$.

Proof. From Lemmas 5.1 and 6.9 we have that $L_{B} L_{C}=(I+P Q)^{-1} P Q$, from which it follows that $P Q=\left(I-L_{B} L_{C}\right)^{-1} L_{B} L_{C}$. Let $\lambda \in \mathbb{C}-\{-1\}$ and define $\mu:=\lambda /(1+\lambda)$, then $\lambda=\mu /(1-\mu)$. We have

$$
\begin{aligned}
\lambda I-P Q & =\frac{\mu}{1-\mu} I-L_{B} L_{C}\left(I-L_{B} L_{C}\right)^{-1} \\
& =\frac{1}{1-\mu}\left[\mu I-(1-\mu) L_{B} L_{C}\left(I-L_{B} L_{C}\right)^{-1}\right] \\
& =\frac{1}{1-\mu}\left[\mu\left(I-L_{B} L_{C}\right)-(1-\mu) L_{B} L_{C}\right]\left(I-L_{B} L_{C}\right)^{-1} \\
& =\frac{1}{1-\mu}\left(\mu I-L_{B} L_{C}\right)\left(I-L_{B} L_{C}\right)^{-1} .
\end{aligned}
$$

This shows that $\lambda \in \sigma(P Q)$ iff $\mu=\lambda /(1+\lambda) \in \sigma\left(L_{B} L_{C}\right)$.

The following lemma gives the desired result.

Lemma 7.2. Let $\left(A_{i}, B_{i}, C_{i}, D\right)$ with $i=1,2$ be two approximately observable input and output stabilizable discrete-time systems. Let $Q_{i}$ and $P_{i}$ denote the optimal cost operators of the system and its dual, respectively. If the two systems have the same input-output map, then the spectra of $P_{1} Q_{1}$ and $P_{2} Q_{2}$ are equal, with the possible exception of zero.

Proof. Denote the gramians of the optimal closed-loop system of $\left(A_{i}, B_{i}, C_{i}, D\right)$ by $L_{B_{i}}$ and $L_{C_{i}}$. Then according to Lemma 7.1, the lemma would be proved if the nonzero elements in the spectrum of $L_{B_{1}} L_{C_{1}}$ equal the nonzero elements in the spectrum of $L_{B_{2}} L_{C_{2}}$. Since the input-output map of both optimal closedloop systems is a normalized factor of the input-output map of the plant by Lemma 5.8 , there exists a unitary $V$ such that $\left[\mathcal{M}_{2} ; \mathcal{N}_{2}\right]=\left[\mathcal{M}_{1} ; \mathcal{N}_{1}\right] V$ by Lemma 5.9. For the Hankel operators of the optimal closed-loop systems this implies $\Gamma_{2}=\Gamma_{1} V$, which implies that $\Gamma_{2} \Gamma_{2}^{*}=\Gamma_{1} \Gamma_{1}^{*}$. Since for arbitrary bounded operators $S$ and $T$ we have that the nonzero elements in the spectrum of $S T$ equal the nonzero elements in the spectrum of $T S$, we have that the nonzero elements in the spectrum of $L_{B} L_{C}=\mathcal{B B}^{*} \mathcal{C}^{*} \mathcal{C}$ equal the nonzero elements in the spectrum of $\Gamma \Gamma^{*}=\mathcal{C B B}^{*} \mathcal{C}^{*}$. This shows that the nonzero elements in the spectrum of $L_{B_{1}} L_{C_{1}}$ equal the nonzero elements in the spectrum of $L_{B_{2}} L_{C_{2}}$.

\section{LQG-balanced realizations}

In this section we prove the existence and some properties of LQG-balanced realizations. We first show that the input-output map of the optimal closedloop system has a Lyapunov-balanced realization. 
Lemma 8.1. Let $(A, B, C, D)$ be an output stabilizable discrete-time system. Then the input-output map of the optimal closed-loop system has an approximately controllable and approximately observable Lyapunov-balanced realization.

Proof. Lemma 5.8 shows that the optimal closed-loop system is input-output stable. This implies that the Hankel operator of the optimal closed-loop system is bounded and Lemma 4.1 then shows that the input-output map of the optimal closed-loop system has a Lyapunov-balanced realization.

From this Lyapunov-balanced realization we can construct a LQG-balanced realization of the plant.

Theorem 8.2. Let $(A, B, C, D)$ be an input and output stabilizable discretetime system. Then the input-output map of the system $(A, B, C, D)$ has a $L Q G$ balanced realization.

Proof. Denote the approximately controllable and approximately observable Lyapunov-balanced realization of the input-output map of the optimal closedloop system by $(\check{A}, \check{B}, \check{C}, \check{D})$. Denote the equal controllability and observability gramians of this realization by $L$. Define

$$
A_{s}:=\check{A}-\check{B}_{\bar{D}}^{-1} \check{C}_{1}, \quad B_{s}:=\check{B} \check{D}_{1}^{-1}, \quad C_{s}:=\check{C}_{2}-\check{D}_{2} \check{D}_{1}^{-1} \check{C}_{1}, \quad D_{s}:=\check{D}_{2} \check{D}_{1}^{-1} .
$$

Then $\left(A_{s}, B_{s}, C_{s}, D_{s}\right)$ is a realization of the input-output map of $(A, B, C, D)$ according to Lemma 5.7. Since the input-output map of the optimal closed-loop system is inner (Corollary 5.8) and $(\check{A}, \check{B}, \check{C}, \check{D})$ is approximately controllable we know from Lemma 5.3 that $\check{B}^{*} L \check{B}+\check{D}^{*} \check{D}=I$ and $\check{B}^{*} L \check{A}+\check{D}^{*} \check{C}=0$. From Lemma 6.4 we see that $L$ is a solution of the CARE of the system $\left(A_{s}, B_{s}, C_{s}, D_{s}\right)$. From Corollary 6.8 we know that $I-L^{2}$ is boundedly invertible, and Lemma 6.6 now tells us that $L\left(I-L^{2}\right)^{-1}$ is a solution of the FARE of the system $\left(A_{s}, B_{s}, C_{s}, D_{s}\right)$. From Lemma 4.3 we know that $\check{A}$ is strongly stable. Using Lemma 3.7 we see that this implies that $L$ is the unique nonnegative self-adjoint solution of the CARE of the system $\left(A_{s}, B_{s}, C_{s}, D_{s}\right)$. Assume that the FARE of the system $\left(A_{s}, B_{s}, C_{s}, D_{s}\right)$ has two nonnegative selfadjoint solutions, $P_{1}$ and $P_{2}$. From Lemma 6.5 we see that $\left(I+P_{1} L\right)^{-1} P_{1}$ and $\left(I+P_{2} L\right)^{-1} P_{2}$ are solutions of the control Lyapunov equation of the optimal closed-loop system of $\left(A_{s}, B_{s}, C_{s}, D_{s}\right)$, which is the balanced realization $(\check{A}, \check{B}, \check{C}, \check{D})$. From Corollary 4.4 we see that $\left(I+P_{1} L\right)^{-1} P_{1}=\left(I+P_{2} L\right)^{-1} P_{2}$, which implies that $P_{1}=P_{2}$. We recall that if a system $\left(A_{1}, B_{1}, C_{1}, D_{1}\right)$ has a solution $Q$ to its CARE and $P$ to its FARE and $S$ is a boundedly invertible operator, then the system $\left(S A S^{-1}, S B, C S^{-1}, D\right)$ has a solution $S^{-*} Q S^{-1}$ to its CARE and $S P S^{*}$ to its FARE. It is easily seen that $I-L^{2}$ is a nonnegative operator (for example, using that the Hankel operator has norm smaller than one), from which it follows that $S:=\left(I-L^{2}\right)^{-1 / 4}$ is well defined. Define $\left(A_{l}, B_{l}, C_{l}, D_{l}\right)=\left(S A_{s} S^{-1}, S B_{s}, C_{s} S^{-1}, D_{s}\right)$. Then the system $\left(A_{l}, B_{l}, C_{l}, D_{l}\right)$ has $L\left(I-L^{2}\right)^{-1 / 2}$ as the unique solution to both its CARE and its FARE.

We now prove that LQG-balanced realizations are essentially unique. 
Lemma 8.3. Let $(A, B, C, D)$ be an approximately controllable and approximately observable $L Q G$-balanced realization. Then all approximately controllable and approximately observable $L Q G$-balanced realizations of the same inputoutput map are given by $\left\{\left(T A T^{-1}, T B, C T^{-1}, D\right): T \in \mathcal{L}(X)\right.$ unitary $\}$.

Proof. It is obvious that the given realizations are indeed LQG balanced; it remains to be proved that these are all approximately controllable and approximately observable LQG-balanced realizations. Let $\left(A_{1}, B_{1}, C_{1}, D_{1}\right)$ and $\left(A_{2}, B_{2}, C_{2}, D_{2}\right)$ be two approximately controllable and approximately observable LQG-balanced realizations of the same input-output map. Let $Q_{1}$ and $Q_{2}$ be the optimal cost operators of the respective systems. Define $S_{i}:=$ $\left(I+Q_{i}^{2}\right)^{1 / 4}$ and $\left(A_{i}^{b}, B_{i}^{b}, C_{i}^{b}, D_{i}^{b}\right)=\left(S_{i} A_{i} S_{i}^{-1}, S_{i} B_{i}, C_{i} S_{i}^{-1}, D_{i}\right)$ for $i=1,2$. Then $\left(A_{i}^{b}, B_{i}^{b}, C_{i}^{b}, D_{i}^{b}\right)$ has $Q_{i}^{b}:=Q_{i}\left(I+Q_{i}^{2}\right)^{-1 / 2}$ as its optimal cost operator and $P_{i}^{b}:=Q_{i}\left(I+Q_{i}^{2}\right)^{1 / 2}$ as the optimal cost operator of its dual system. Let $\left(\check{A}_{i}^{b}, \check{B}_{i}^{b}, \check{C}_{i}^{b}, \check{D}_{i}^{b}\right)$ be the optimal closed-loop system of $\left(A_{i}^{b}, B_{i}^{b}, C_{i}^{b}, D_{i}^{b}\right)$. Using Lemmas 5.1 and 6.9 we see that they are Lyapunov-balanced realizations with gramians $L_{i}:=Q_{i}\left(I+Q_{i}^{2}\right)^{-1 / 2}$. According to Corollary 5.8 the inputoutput maps of $\left(\check{A}_{1}^{b}, \check{B}_{1}^{b}, \check{C}_{1}^{b}, \check{D}_{1}^{b}\right)$ and $\left(\check{A}_{2}^{b}, \check{B}_{2}^{b}, \check{C}_{2}^{b}, \check{D}_{2}^{b}\right)$ are normalized factors of the input-output map of $\left(A_{1}, B_{1}, C_{1}, D_{1}\right)$ (which equals the input-output map of $\left.\left(A_{2}, B_{2}, C_{2}, D_{2}\right)\right)$. By Lemma 5.9 there exists an operator $V$ such that $\left[\mathcal{M}_{2} ; \mathcal{N}_{2}\right]=\left[\mathcal{M}_{1} ; \mathcal{N}_{1}\right] V$. It is easily seen that $\left(\check{A}_{1}^{b}, \check{B}_{1}^{b} V, \check{C}_{1}^{b}, \check{D}_{1}^{b} V\right)$ is a Lyapunov-balanced realization of $\left[\mathcal{M}_{1} ; \mathcal{N}_{1}\right] V=\left[\mathcal{M}_{2} ; \mathcal{N}_{2}\right]$. From Lemma 6.2 it follows that both $\left(\check{A}_{1}^{b}, \check{B}_{1}^{b} V, \check{C}_{1}^{b}, \check{D}_{1}^{b} V\right)$ and $\left(\check{A}_{2}^{b}, \check{B}_{2}^{b}, \check{C}_{2}^{b}, \check{D}_{2}^{b}\right)$ are approximately controllable and approximately observable Lyapunov-balanced realizations of the input-output map $\left[\mathcal{M}_{2} ; \mathcal{N}_{2}\right]$. From Lemma 4.1 it follows that there exists a unitary $T$ such that

$$
\left(T \check{A}_{1}^{b} T^{-1}, T \check{B}_{1}^{b} V, \check{C}_{1}^{b} T^{-1}, \check{D}_{1}^{b} V\right)=\left(\check{A}_{2}^{b}, \check{B}_{2}^{b}, \check{C}_{2}^{b}, \check{D}_{2}^{b}\right) .
$$

Using (18) for $i=1,2$ we see that

$$
\left(T A_{1}^{b} T^{-1}, T B_{1}^{b}, C_{1}^{b} T^{-1}, D_{1}^{b}\right)=\left(A_{2}^{b}, B_{2}^{b}, C_{2}^{b}, D_{2}^{b}\right) .
$$

It now follows that

$$
\left(S_{2}^{-1} T S_{1} A_{1} S_{1}^{-1} T^{-1} S_{2}, S_{2}^{-1} T S_{1} B_{1}, C_{1} S_{1}^{-1} T^{-1} S_{2}, D_{1}\right)=\left(A_{2}, B_{2}, C_{2}, D_{2}\right) .
$$

To complete the proof that $\left(A_{1}, B_{1}, C_{1}, D_{1}\right)$ and $\left(A_{2}, B_{2}, C_{2}, D_{2}\right)$ are unitarily equivalent we prove that $S_{2}^{-1} T S_{1}=T$ or equivalently $T S_{1} T^{-1}=S_{2}$. Since $\left(I+Q_{i}^{2}\right)^{-1}=I-\left[Q_{i}\left(I+Q_{i}^{2}\right)^{-1 / 2}\right]^{2}=I-L_{i}^{2}$ we have $S_{i}=\left(I+Q_{i}^{2}\right)^{-1 / 4}=$ $\left(I-L_{i}^{2}\right)^{1 / 4}$ and since $L_{2}=T L_{1} T^{-1}$ we then have

$$
S_{2}=\left(I-L_{2}^{2}\right)^{1 / 4}=T\left(I-L_{1}^{2}\right)^{1 / 4} T^{-1}=T S_{1} T^{-1} .
$$

This proves that all approximately controllable and approximately observable LQG-balanced realizations of the same input-output map are unitarily equivalent. 
The following lemma states what the proof of Theorem 8.2 already indicated, namely, that the filter and control Riccati equations of an approximately controllable and approximately observable LQG-balanced realization have unique nonnegative self-adjoint solutions.

Lemma 8.4. Let $(A, B, C, D)$ be an approximately controllable and approximately observable $L Q G$-balanced realization. Then both the CARE (5) and the FARE (9) of $(A, B, C, D)$ have a unique nonnegative self-adjoint solution.

Proof. As in the proof of Lemma 8.3 we construct the approximately controllable and approximately observable realization $\left(A^{b}, B^{b}, C^{b}, D^{b}\right)$ and the approximately controllable and approximately observable Lyapunov-balanced realization $\left(\check{A}^{b}, \check{B}^{b}, \check{C}^{b}, \check{D}^{b}\right)$. From Lemma 4.3 we know that $\check{A}^{b}$ is strongly stable. Lemma 3.7 shows that the CARE of $\left(A^{b}, B^{b}, C^{b}, D^{b}\right)$ has a unique nonnegative self-adjoint solution. Obviously this implies that the CARE of $(A, B, C, D)$ has a unique nonnegative self-adjoint solution. From Lemma 4.4 we know that the control Lyapunov equation of $\left(\check{A}^{b}, \check{B}^{b}, \check{C}^{b}, \check{D}^{b}\right)$ has a unique nonnegative selfadjoint solution. As in the proof of Theorem 8.2, it follows that the FARE of $\left(A^{b}, B^{b}, C^{b}, D^{b}\right)$ and hence the FARE of $(A, B, C, D)$ has a unique nonnegative self-adjoint solution.

\section{Conclusions}

We have proved the existence of LQG-balanced realizations for the class of transfer functions that are analytic on some disc centered at the origin and have a (infinite-dimensional) realization that is both input and output stabilizable. We have also proved that approximately controllable and approximately observable LQG-balanced realizations are essentially unique and that the Riccati equations of approximately controllable and approximately observable LQG-balanced realizations have unique nonnegative self-adjoint solutions. Analogous continuoustime results and error bounds for truncations of LQG-balanced realizations will be given elsewhere.

\section{Appendix}

\subsection{Miscellaneous results on Riccati operators}

Lemma 10.1. Let $P$ and $Q$ be nonnegative self-adjoint operators. Define

$$
\begin{gathered}
A_{P}:=A-\left(B D^{*}+A P C^{*}\right)\left(R+C P C^{*}\right)^{-1} C, \\
A_{Q}:=A-B\left(S+B^{*} Q B\right)^{-1}\left(D^{*} C+B^{*} Q A\right), \\
\underline{A}:=A-B S^{-1} D^{*} C .
\end{gathered}
$$


where $S:=I+D^{*} D$ and $R:=I+D D^{*}$. Then

$$
\begin{gathered}
A_{P}\left(I+P C^{*} R^{-1} C\right)=\underline{A}=\left(I+B S^{-1} B^{*} Q\right) A_{Q}, \\
A_{Q}=\left(I+B S^{-1} B^{*} Q\right)^{-1} A_{P}\left(I+P C^{*} R^{-1} C\right), \\
A_{P}=\left(I+B S^{-1} B^{*} Q\right) A_{Q}\left(I+P C^{*} R^{-1} C\right)^{-1} .
\end{gathered}
$$

Proof. We prove that $A_{P}\left(I+P C^{*} R^{-1} C\right)=\underline{A}$. The equality $\underline{A}=\left(I+B S^{-1} B^{*} Q\right) A_{Q}$ is proved similarly. By writing out $A_{P}$ in full we have

$$
\begin{aligned}
& A_{P}\left(I+P C^{*} R^{-1} C\right) \\
& \quad=A\left(I+P C^{*} R^{-1} C\right)-\left(B D^{*}+A P C^{*}\right)\left(R+C P C^{*}\right)^{-1} C\left(I+P C^{*} R^{-1} C\right) \\
& \quad=A\left(I+P C^{*} R^{-1} C\right)-\left(B D^{*}+A P C^{*}\right)\left(R+C P C^{*}\right)^{-1}\left(R+C P C^{*}\right) R^{-1} C \\
& \quad=A+A P C^{*} R^{-1} C-\left(B D^{*}+A P C^{*}\right) R^{-1} C=A-B D^{*} R^{-1} C \\
& =A-B S^{-1} D^{*} C,
\end{aligned}
$$

since $D^{*} R^{-1}=S^{-1} D^{*}$. This completes the proof of (28). Equations (29) and (30) easily follow from (28).

Note that in the above lemma we have not assumed that $P$ and $Q$ are solutions of the Riccati equations.

We now prove that the Riccati equations can be written in several different but equivalent versions.

Lemma 10.2. $\quad$ 1. $P$ is a nonnegative self-adjoint solution of

$$
A_{P} P\left(I+C^{*} R^{-1} C P\right) A_{P}^{*}-P+B S^{-1} B^{*}=0,
$$

where $A_{P}$ is defined by (25), iff it is a nonnegative self-adjoint solution of

$$
\underline{A} P\left(I+C^{*} R^{-1} C P\right)^{-1} \underline{A}^{*}-P+B S^{-1} B^{*}=0,
$$

where $\underline{A}$ is defined by $(27)$.

2. $P$ is a nonnegative self-adjoint solution of (31) iff it is a nonnegative selfadjoint solution of the FARE (9).

3. $Q$ is a nonnegative self-adjoint solution of

$$
A_{Q}^{*}\left(I+Q B S^{-1} B^{*}\right) Q A_{Q}-Q+C^{*} R^{-1} C=0,
$$

where $A_{Q}$ is defined by (26), iff it is a nonnegative self-adjoint solution of

$$
\underline{A}^{*} Q\left(I+B S^{-1} B^{*} Q\right)^{-1} \underline{A}-Q+C^{*} R^{-1} C=0,
$$

where $\underline{A}$ is defined by (27).

4. $Q$ is a nonnegative self-adjoint solution of (33) iff it is a nonnegative selfadjoint solution of the CARE (5). 
Proof. We shall prove the equivalence of the filter equations; the equivalence of the control equations is similar.

1. The equations (31) and (32) are equivalent iff the following holds:

$$
\underline{A} P\left(I+C^{*} R^{-1} C P\right)^{-1} \underline{A}^{*}=A_{P} P\left(I+C^{*} R^{-1} C P\right) A_{P}^{*} .
$$

We use Lemma 10.1 (which tells us that $\underline{A}=A_{P}\left(I+P C^{*} R^{-1} C\right)$ ) to write the left-hand side of (35) as

$$
A_{P}\left(I+P C^{*} R^{-1} C\right) P\left(I+C^{*} R^{-1} C P\right)^{-1}\left(I+C^{*} R^{-1} C P\right) A_{P}^{*},
$$

which is indeed equal to the right-hand side of (35).

2. To prove the equivalence of (31) and (9) we substitute in (31) for $A_{P}$ from (25) and for $\left(I+C^{*} R^{-1} C P\right) A_{P}^{*}$, we substitute $\underline{A}^{*}$ (using (28)) and then substitute (27) for $\underline{A}$. We then get

$$
\left(A-\left(B D^{*}+A P C^{*}\right)\left(R+C P C^{*}\right)^{-1} C\right) P\left(A^{*}-C^{*} D S^{-1} B^{*}\right)-P+B S^{-1} B^{*}=0 .
$$

Rewriting this gives

$$
\begin{aligned}
A P A^{*}-P+B B^{*} & =\left(B D^{*}+A P C^{*}\right)\left(R+C P C^{*}\right)^{-1} C P A^{*} \\
& -\left(B D^{*}+A P C^{*}\right)\left(R+C P C^{*}\right)^{-1} C P C^{*} D S^{-1} B^{*}+A P C^{*} D S^{-1} B^{*} \\
& -B S^{-1} B^{*}+B B^{*} .
\end{aligned}
$$

We now focus on the last two lines of this last equation. We note that $I-S^{-1}=$ $D^{*} D S^{-1}$ and we can thus rewrite these last two lines as

$-\left(B D^{*}+A P C^{*}\right)\left(R+C P C^{*}\right)^{-1} C P C^{*} D S^{-1} B^{*}+A P C^{*} D S^{-1} B^{*}+B D^{*} D S^{-1} B^{*}$,

and this can be rewritten as

$$
\left(B D^{*}+A P C^{*}\right)\left(R+C P C^{*}\right)^{-1}\left[-C P C^{*}+R+C P C^{*}\right] D S^{-1} B^{*} .
$$

Noting that $R D S^{-1}=D$, we see that this is equal to

$$
\left(B D^{*}+A P C^{*}\right)\left(R+C P C^{*}\right)^{-1} D B^{*} .
$$

This completes the proof of the equivalence of (31) and (9).

We now show that the known relationship between $A_{Q}$ and $A_{P}$ extends to the infinite-dimensional case.

Lemma 10.3. Let $(A, B, C, D)$ be an input and output stabilizable discrete-time system, let $Q$ be a nonnegative self-adjoint solution of its CARE (5), and let $P$ be a nonnegative self-adjoint solution of its FARE (9). Define $A_{P}$ and $A_{Q}$ by (25) and (26), respectively. Then

$$
(I+P Q) A_{Q}=A_{P}(I+P Q) .
$$


Proof. We use FARE (31) to write

$$
P=A_{P} P\left(I+C^{*} R^{-1} C P\right) A_{P}^{*}+B S^{-1} B^{*},
$$

which leads to

$$
I+P Q=I+A_{P} P\left(I+C^{*} R^{-1} C P\right) A_{P}^{*} Q+B S^{-1} B^{*} Q
$$

and so

$$
(I+P Q) A_{Q}=\left(I+B S^{-1} B^{*} Q\right) A_{Q}+A_{P} P\left(I+C^{*} R^{-1} C P\right) A_{P}^{*} Q A_{Q} .
$$

We use (29) to write the right-hand side as

$$
A_{P}\left(I+P C^{*} R^{-1} C\right)+A_{P} P\left(I+C^{*} R^{-1} C P\right) A_{P}^{*} Q A_{Q} .
$$

Rearranging gives

$$
A_{P}+A_{P} P\left[C^{*} R^{-1} C+\left(I+C^{*} R^{-1} C P\right) A_{P}^{*} Q A_{Q}\right],
$$

and using (29) again we obtain

$$
A_{P}+A_{P} P\left[C^{*} R^{-1} C+A_{Q}^{*}\left(I+Q B S^{-1} B^{*}\right) Q A_{Q}\right] .
$$

According to CARE (33), the term in square brackets equals $Q$. So the above is equal to $A_{P}(I+P Q)$.

We now prove a relation concerning the difference of two solutions of a Riccati equation.

Lemma 10.4. Let $(A, B, C, D)$ be an output stabilizable discrete-time system and let $Q_{1}$ and $Q_{2}$ be nonnegative self-adjoint solutions of its CARE (5). Define $A_{Q_{1}}$ and $A_{Q_{2}}$ similarly to (26). Then

$$
Q_{1}-Q_{2}=A_{Q_{2}}^{*}\left(Q_{1}-Q_{2}\right) A_{Q_{1}} .
$$

Proof. Subtract the form (33) of the CARE for $Q_{1}$ and $Q_{2}$ to obtain

$$
Q_{1}-Q_{2}=A_{Q_{1}}^{*}\left(I+Q_{1} B S^{-1} B^{*}\right) Q_{1} A_{Q_{1}}-A_{Q_{2}}^{*}\left(I+Q_{2} B S^{-1} B^{*}\right) Q_{2} A_{Q_{2}} .
$$

According to Lemma 10.1 (say with $P=I$ ) we have

$$
\begin{aligned}
A_{Q_{2}} & =\left(I+B S^{-1} B^{*} Q_{2}\right)^{-1} A_{P}\left(I+P C^{*} R^{-1} C\right) \\
& =\left(I+B S^{-1} B^{*} Q_{2}\right)^{-1}\left(I+B S^{-1} B^{*} Q_{1}\right) A_{Q_{1}} .
\end{aligned}
$$

Combining (36) and (37) we obtain

$$
\begin{aligned}
Q_{1}-Q_{2} & =A_{Q_{2}}^{*}\left(I+Q_{2} B S^{-1} B^{*}\right) Q_{1} A_{Q_{1}}-A_{Q_{2}}^{*} Q_{2}\left(I+B S^{-1} B^{*} Q_{1}\right) A_{Q_{1}} \\
& =A_{Q_{2}}^{*}\left(Q_{1}-Q_{2}\right) A_{Q_{1}} .
\end{aligned}
$$




\subsection{Proofs for section 6}

In this section we prove the relationships between the CARE and the FARE of a system and the control and observation Lyapunov equations of its CARE closed-loop system (Lemmas 6.3, 6.4, 6.5, and 6.6).

Lemma 10.5. Suppose that the system $\left(\check{A}, \check{B},\left[\check{C}_{1} ; \check{C}_{2}\right],\left[\check{D}_{1} ; \check{D}_{2}\right]\right)$ is such that $\check{D}_{1}$ is boundedly invertible and that there exists a nonnegative self-adjoint operator $V$ such that

$$
\check{B}^{*} V \check{A}+\check{D}^{*} \check{C}=0 .
$$

Define the system $(A, B, C, D)$ by (18) and $S:=I+D^{*} D$ and $R:=I+D D^{*}$. Then

1. $\check{A}=A-B\left(S+B^{*} V B\right)^{-1}\left(D^{*} C+B^{*} V A\right)$ and

2. $\check{A}^{*} V \check{A}-V+\check{C}^{*} \check{C}=\check{A}^{*}\left(I+V B S^{-1} B^{*}\right) V \check{A}-V+C^{*} R^{-1} C$.

Proof. We first prove the equality

$$
S \check{C}_{1}=-\left(B^{*} V \check{A}+D^{*} C\right) .
$$

From (38) and (18) we obtain

$$
\check{D}_{1}^{*} B^{*} V \check{A}+\check{D}_{1}^{*} \check{C}_{1}+\check{D}_{2}^{*} \check{C}_{2}=0 .
$$

Thus

$$
\check{C}_{1}=-B^{*} V \check{A}-D^{*} \check{C}_{2}=-B^{*} V \check{A}-D^{*}\left(C+D \check{C}_{1}\right)
$$

and this yields (39):

$$
S \check{C}_{1}=\left(I+D^{*} D\right) \check{C}_{1}=-B^{*} V \check{A}-D^{*} C .
$$

We now prove the first equality stated in the lemma. We take the equality just proved (39) and substitute $\check{A}=A+B \check{C}_{1}$ to obtain

$$
S \check{C}_{1}=-\left(B^{*} V\left(A+B \check{C}_{1}\right)+D^{*} C\right) .
$$

Thus

$$
\left(S+B^{*} V B\right) \check{C}_{1}=-\left(B^{*} V A+D^{*} C\right) .
$$

We now solve for $\check{C}_{1}$ and substitute to obtain

$$
\check{A}=A+B \check{C}_{1}=A-B\left(S+B^{*} V B\right)^{-1}\left(B^{*} V A+D^{*} C\right) .
$$

We now prove the equality

$$
\check{C}^{*} \check{C}=\check{A}^{*} V B S^{-1} B^{*} V \check{A}+C^{*} R^{-1} C .
$$

We have

$$
\check{C}^{*} \check{C}=\check{C}_{1}^{*} \check{C}_{1}+\check{C}_{2}^{*} \check{C}_{2}
$$

and substituting for $\check{C}_{2}$ from (18) gives

$$
\check{C}^{*} \check{C}=\check{C}_{1}^{*} \check{C}_{1}+\left(C+D \check{C}_{1}\right)^{*}\left(C+D \check{C}_{1}\right) .
$$

Finally, substituting for $\check{C}_{1}$ from (39) and simplifying gives the result. The second equality stated in the lemma follows easily from (40). 
Proof of Lemma 6.3. We only have to prove (23). Noting (22), we apply Lemma 10.5 with $V:=Q$ to the CARE closed-loop system. Since $A$ defined by (15) equals $A_{Q}$ defined by (26), we have

$$
\check{A}^{*} Q \check{A}-Q+\check{C}^{*} \check{C}=A_{Q}^{*}\left(I+Q B S^{-1} B^{*}\right) Q A_{Q}-Q+C^{*} R^{-1} C .
$$

Since $Q$ is a solution of the CARE (5) of $(A, B, C, D)$ the right-hand side of this equation is zero by Lemma 10.2 and we have shown (23).

Proof of Lemma 6.4. By assumption, there exists a nonnegative self-adjoint $Q$ such that $\check{B}^{*} Q \breve{A}+\check{D}^{*} \check{C}=0$. We apply Lemma 10.5 with $V:=Q$ to obtain $\check{A}=A_{Q}$ given by $(26)$ and

$$
\check{A}^{*} Q \check{A}-Q+\check{C}^{*} \check{C}=A_{Q}^{*}\left(I+Q B S^{-1} B^{*}\right) Q A_{Q}-Q+C^{*} R^{-1} C .
$$

The left-hand side of this equation is zero since by assumption $Q$ satisfies (23). This proves that the right-hand side is zero, and Lemma 10.2 now shows that $Q$ is a solution of the CARE of the system $(A, B, C, D)$.

Lemma 10.6. Let a system $\left(\check{A}, \check{B},\left[\check{C}_{1} ; \check{C}_{2}\right],\left[\check{D}_{1} ; \check{D}_{2}\right]\right)$ with $\check{D}_{1}$ boundedly invertible be given and assume that a nonnegative self-adjoint operator $V$ exists such that

$$
\check{B}^{*} V \check{B}+\check{D}^{*} \check{D}=I .
$$

Define the system $(A, B, C, D)$ by (18). Then we have

1. $B^{*} V B+S=\check{D}_{1}^{-*} \check{D}_{1}^{-1}$ and

2. $\check{B} \check{B}^{*}\left(I+V B S^{-1} B^{*}\right)=B S^{-1} B^{*}$.

Proof. 1. The given equation for $V$ translates to

$$
\check{D}_{1}^{*} B^{*} V B \check{D}_{1}+\check{D}_{1}^{*} \check{D}_{1}+\check{D}_{1}^{*} D^{*} D \check{D}_{1}=I,
$$

and multiplying from the left with $\check{D}_{1}^{-*}$ and from the right with $\check{D}_{1}^{-1}$ gives the result.

2. The first equality implies that $\left(S+B^{*} V B\right)^{-1}=\check{D}_{1} \check{D}_{1}^{*}$ and so $B(S+$ $\left.B^{*} V B\right)^{-1} B^{*}$

$=\check{B} \check{B}^{*}$. Hence

$$
\begin{aligned}
\check{B} \check{B}^{*}\left(I+V B S^{-1} B^{*}\right) & =B\left(S+B^{*} V B\right)^{-1} B^{*}\left(I+V B S^{-1} B^{*}\right) \\
& =B\left(S+B^{*} V B\right)^{-1}\left(S+B^{*} V B\right) S^{-1} B^{*}=B S^{-1} B^{*}
\end{aligned}
$$

which proves the second equality.

We now prove Lemma 6.5.

Proof of Lemma 6.5. We remark that $L=(I+P Q)^{-1} P=P(I+Q P)^{-1}$ and so we have to show that

$$
\check{A} P(I+Q P)^{-1} \check{A}^{*}-P(I+Q P)^{-1}+\check{B} \check{B}^{*}=0 .
$$


Recalling that $\check{A}=A_{Q}$ from (15) and (26) and using Lemmas 10.1 (29) and 10.3 we can substitute $\check{A}=\left(I+B S^{-1} B^{*} Q\right)^{-1} A_{P}\left(I+P C^{*} R^{-1} C\right)$ and $(I+$ $Q P)^{-1} \check{A}^{*}=A_{P}^{*}(I+Q P)^{-1}$ to obtain for the left-hand side of (41)

$$
\begin{aligned}
(I & \left.+B S^{-1} B^{*} Q\right)^{-1} A_{P}\left(I+P C^{*} R^{-1} C\right) P A_{P}^{*}(I+Q P)^{-1}-P(I+Q P)^{-1}+\check{B} \check{B}^{*} \\
& =\left(I+B S^{-1} B^{*} Q\right)^{-1}\left[A_{P}\left(I+P C^{*} R^{-1} C\right) P A_{P}^{*}-\left(I+B S^{-1} B^{*} Q\right) P\right. \\
& \left.+\left(I+B S^{-1} B^{*} Q\right)^{-1} \check{B} \check{B}^{*}(I+Q P)\right](I+Q P)^{-1} .
\end{aligned}
$$

We now apply Lemma 10.6 with $V:=Q$ to obtain $\left(I+B S^{-1} B^{*} Q\right)^{-1} \check{B} \check{B}^{*}=$ $B S^{-1} B^{*}$, and so we obtain for the left-hand side of (41)

$$
\begin{aligned}
\left(I+B S^{-1} B^{*} Q\right)^{-1}\left[A_{P}\left(I+P C^{*} R^{-1} C\right) P A_{P}^{*}\right. & -\left(I+B S^{-1} B^{*} Q\right) P \\
& \left.+B S^{-1} B^{*}(I+Q P)\right](I+Q P)^{-1} .
\end{aligned}
$$

The term in square brackets is zero according to the FARE (31) and we have proved (41).

Proof of Lemma 6.6. We first recall from the proof of Lemma 6.4 that $\check{A}=A_{Q}$, where $A_{Q}$ is defined by (26), and that (30) holds with $A_{Q}=\check{A}$. Define $P:=L(I-Q L)^{-1}$ and define $A_{P}$ by (25). The second step in the proof is establishing the identity

$$
(I-L Q) A_{P}=\check{A}(I-L Q)
$$

or by (30) the equivalent identity

$$
(I-L Q)\left(I+B S^{-1} B^{*} Q\right) \check{A}=\check{A}(I-L Q)\left(I+P C^{*} R^{-1} C\right) .
$$

Since $P=L(I-Q L)^{-1}=(I-L Q)^{-1} L$ the right-hand side of (43) is equal to

$$
\check{A}-\check{A} L Q+\check{A} L C^{*} R^{-1} C \text {. }
$$

We substitute $Q-C^{*} R^{-1} C=\check{A}^{*}\left(I+Q B S^{-1} B^{*}\right) Q \check{A}$ (this identity holds because $Q$ is a solution of the CARE; see (33)) to obtain for the right-hand side of (43)

$$
\check{A}-\check{A} L \check{A}^{*}\left(I+Q B S^{-1} B^{*}\right) Q \check{A} .
$$

The control Lyapunov equation tells us that $\check{A} L \check{A}^{*}=L-\check{B} \check{B}^{*}$ and so the right-hand side of (43) is equal to

$$
\check{A}-L\left(I+Q B S^{-1} B^{*}\right) Q \check{A}+\check{B} \check{B}^{*}\left(I+Q B S^{-1} B^{*}\right) Q \check{A} .
$$

Substituting $\check{B} \check{B}^{*}\left(I+Q B S^{-1} B^{*}\right)=B S^{-1} B^{*}$ from Lemma 10.6 with $V=Q$ we obtain for the right-hand side of (43)

$$
\check{A}-L\left(I+Q B S^{-1} B^{*}\right) Q \check{A}+B S^{-1} B^{*} Q \check{A},
$$

which is equal to the left-hand side of (43). This proves (42). We now make the third and last step of the proof that $P$ is a solution of the FARE. We start with the control Lyapunov equation

$$
\check{A} L \check{A}^{*}-L+\check{B} \check{B}^{*}=0
$$


and substitute $\check{A}=(I-L Q) A_{P}(I-L Q)^{-1}$ from $(42)$ and $\check{A}^{*}=\left(I+C^{*} R^{-1} C P\right) A_{P}^{*}(I+$ $\left.Q B S^{-1} B^{*}\right)^{-1}$ from (30) to obtain

$(I-L Q) A_{P}(I-L Q)^{-1} L\left(I+C^{*} R^{-1} C P\right) A_{P}^{*}\left(I+Q B S^{-1} B^{*}\right)^{-1}-L+\check{B} \check{B}^{*}=0$.

We multiply by $(I-L Q)^{-1}$ from the left and by $\left(I+Q B S^{-1} B^{*}\right)$ from the right to obtain

$A_{P} P\left(I+C^{*} R^{-1} C P\right) A_{P}^{*}-P\left(I+Q B S^{-1} B^{*}\right)+(I-L Q)^{-1} \check{B} \check{B}^{*}\left(I+Q B S^{-1} B^{*}\right)=0$.

We again use the fact that $\check{B} \check{B}^{*}\left(I+Q B S^{-1} B^{*}\right)=B S^{-1} B^{*}$ to obtain

$$
A_{P} P\left(I+C^{*} R^{-1} C P\right) A_{P}^{*}-P-P Q B S^{-1} B^{*}+(I-L Q)^{-1} B S^{-1} B^{*}=0 .
$$

Using that $P=(I-L Q)^{-1} L$ we see that the sum of the two last terms of the left-hand side of (44) equals $B S^{-1} B^{*}$. This proves that $P$ is a solution of the equivalent version (31) of the FARE.

\section{References}

[1] R.F. Curtain and H.J. Zwart, An Introduction to Infinite-Dimensional Linear Systems Theory, Springer-Verlag, New York, 1995.

[2] P.A. Fuhrmann and R. Ober, A functional approach to $L Q G$ balancing, Internat. J. Control, 57 (1993), pp. 627-741.

[3] A. Halanay and V. Ionescu, Time Varying Discrete Linear Systems, Birkhäuser, Basel, 1994.

[4] J. Hoffmann, D. Prätzel-Wolters, and E. Zerz, A balanced canonical form for discrete-time minimal systems using characteristic maps, Linear Algebra Appl., 277 (1998), pp. 63-81.

[5] E.A. Jonckheere And L.M. Silverman, A new set of invariants for linear systems-application to reduced order compensator design, IEEE Trans. Automat. Control., 28 (1983), pp. 953-964.

[6] D.G. Meyer and G.F. Franklin, A connection between normalized coprime factorizations and linear quadratic regulator theory, IEEE Trans. Automat. Control, 32 (1987), pp. 227-228.

[7] B.C. Moore, Principal component analysis in linear systems: Controllability, observability, and model reduction, IEEE Trans. Automat. Control, 26 (1981), pp. 17-32.

[8] D. Mustafa and K. Glover, Minimum Entropy Control, SpringerVerlag, Berlin, 1990. 
[9] R.J. Ober And D.C. McFarlane, Balanced canonical forms for minimal systems: A normalized coprime factor approach, Linear Algebra Appl., 122 (1989), pp. 23-64.

[10] R. OBer AND Y. Wu, Asymptotic stability of infinite-dimensional discrete-time balanced realizations, SIAM J. Control Optim., 31 (1993), pp. 1321-1339.

[11] A.J. Sasane, Hankel Norm Approximation for Infinite-Dimensional Systems, Springer-Verlag, Berlin, 2002.

[12] O.J. Staffans, Well-Posed Linear Systems, Encyclopedia Math. Appl. 103, Cambridge University Press, Cambridge, UK, to appear.

[13] E.I. VERRIEST, Low sensitivity design and optimal order reduction for the $L Q G$-problem, in Proceedings of the 24th Midwest Symposium on Circuit Systems, Albuquerque, NM, 1981, pp. 365-369.

[14] E.I. VerRIEST, Suboptimal LQG-design via balanced realizations, in Proceedings of the 20th IEEE Conference on Decision and Control, San Diego, CA, 1981, pp. 686-687.

[15] N.J. Young, Balanced realizations in infinite dimensions, Oper. Theory Adv. Appl., 19 (1986), pp. 449-471.

[16] N.J. Young, Balanced, normal, and intermediate realizations of nonrational transfer functions, IMA J. Math. Control Inform., 3 (1986), pp. 4358.

[17] K. Zhou and J.C. Doyle, Essentials of Robust Control, Prentice-Hall, Upper Saddle River, NJ, 1998. 